\title{
Comparative genomic analyses reveal broad diversity in botulinum-toxin- producing Clostridia
}

\author{
Charles H. D. Williamson", Jason W. Sahl', Theresa J. Smith², Gary Xie” ${ }^{3}$ Brian T. Foley ${ }^{4}$, Leonard A. Smith ${ }^{5}$, \\ Rafael A. Fernández ${ }^{6}$, Miia Lindström ${ }^{7}$, Hannu Korkeala ${ }^{7}$, Paul Keim ${ }^{1 *}$, Jeffrey Foster ${ }^{1,8^{*}}$ and Karen Hill $\left.\right|^{3^{*}}$
}

\begin{abstract}
Background: Clostridium botulinum is a diverse group of bacteria characterized by the production of botulinum neurotoxin. Botulinum neurotoxins are classified into serotypes (BoNT/A-G), which are produced by six species/ Groups of Clostridia, but the genetic background of the bacteria remains poorly understood. The purpose of this study was to use comparative genomics to provide insights into the genetic diversity and evolutionary history of bacteria that produce the potent botulinum neurotoxin.
\end{abstract}

Results: Comparative genomic analyses of over 170 Clostridia genomes, including our draft genome assemblies for 59 newly sequenced Clostridia strains from six continents and publicly available genomic data, provided in-depth insights into the diversity and distribution of BoNT-producing bacteria. These newly sequenced strains included Group I and II strains that express BoNT/A,/B,/E, or/F as well as bivalent strains. BoNT-producing Clostridia and closely related Clostridia species were delineated with a variety of methods including 165 rRNA gene, concatenated marker genes, core genome and concatenated multi-locus sequencing typing (MLST) gene phylogenies that related whole genome sequenced strains to publicly available strains and sequence types. These analyses illustrated the phylogenetic diversity in each Group and the diversity of genomic backgrounds that express the same toxin type or subtype. Comparisons of the botulinum neurotoxin genes did not identify novel toxin types or variants.

Conclusions: This study represents one of the most comprehensive analyses of whole genome sequence data for Group I and II BoNT-producing strains. Read data and draft genome assemblies generated for 59 isolates will be a resource to the research community. Core genome phylogenies proved to be a powerful tool for differentiating BoNT-producing strains and can provide a framework for the study of these bacteria. Comparative genomic analyses of Clostridia species illustrate the diversity of botulinum-neurotoxin-producing strains and the plasticity of the genomic backgrounds in which bont genes are found.

Keywords: Clostridium botulinum, Botulinum neurotoxin, Whole genome sequence, Comparative genomics

\section{Background}

Clostridium botulinum encompasses diverse anaerobic, spore-forming bacteria that are defined by the production of one, two or three botulinum neurotoxins (BoNTs) [1]. The botulinum neurotoxin produces a flaccid paralysis known as botulism that affects humans, other mammals, birds and fish [2]. There are seven serotypes of botulinum

\footnotetext{
* Correspondence: Paul.Keim@nau.edu; Jeff.Foster@unh.edu; khill@lanl.gov ${ }^{1}$ Center for Microbial Genetics and Genomics, Northern Arizona University, Flagstaff, AZ 86011, USA

${ }^{3}$ Bioscience Division, Los Alamos National Laboratory, Los Alamos, NM 87545, USA Full list of author information is available at the end of the article
}

neurotoxins, BoNT/A-G, produced by six known Groups of Clostridia [3]. Recently, sequencing of an infant botulism isolate has also identified an unusual toxin type (BoNT/H or F/A) that is produced in combination with BoNT/B [4, 5]. Group I includes BoNT/A,/B and/F-producing strains; Group II includes BoNT/B,/E and/F-producing strains; Group III includes BoNT/C and/D-producing strains; Group IV includes the BoNT/G-producing C. argentinense strains; Group V includes the BoNT/F-producing $C$. baratii strains; and Group VI includes the BoNT/Eproducing C. butyricum strains [6]. BoNT/A-G are 
35-70 \% different (amino acid identity) from each other and can vary within a toxin type [3]. The variants within a serotype are described by a numerical designation following the toxin such as A1, A2, A3, etc. Thus, BoNT-producing bacteria are members of at least four different bacterial species, as well as several well-defined subclades, that contain a large diversity of toxin types.

Numerous recombination events between toxins have been documented $[1,7,8]$, and bivalent toxin combinations within the same strain have been identified [3]. Strains in different Groups can produce the same toxin (e.g. Group I, II and V strains produce BoNT/F). Horizontal gene transfer of the toxin gene between strains in the six Groups via toxin gene associations with transposases such as insertion sequence (IS) elements, recombinases, the acquisition of plasmids or infection by phage [9-11] is supported by incongruent topologies between the bont gene and 16S rRNA gene phylogenies as well as the presence of the same toxin subtype on the chromosome in some strains and on plasmids in other strains $[3,6]$. Recombination among toxins and horizontal gene transfer between different species and/or Groups therefore allow for substantial variation in botulinum neurotoxins and in the genomic backgrounds in which the same toxin type or subtype are found. Thus, capturing data for both the genomic background as well as the toxin type provides valuable information about the diversity within BoNT-producing species/Groups and how this genetic and phenotypic variation is generated.

Group designations were initially established based upon biochemical and microbiological attributes of the bacteria, though the Group designations and the genetic variation of the bacteria and the toxin have been increasingly investigated using different molecular techniques [12]. The first genomic sequence of a BoNT-producing strain, C. botulinum ATCC 3502, was used to develop microarrays to query Group I strains $[13,14]$. Later the whole genomic sequence of Eklund 17B was used to construct a DNA microarray and query Group II strains [15]. These microarray studies identified diversity in the bacteria that express the botulinum toxin and identified clades of bacteria within their collections that shared common genes. Amplified fragment-length polymorphism (AFLP) analysis has been used to examine the diversity of Group I [7] and II strains [16] and to identify the complexity of 1090 strains of neurotoxin-producing Clostridia primarily from California infant botulism cases [17]. Pulsed-field gel electrophoresis (PFGE) has been used to examine BoNT-producing strains $[18,19]$, including the determination of toxin gene cluster variation and location (plasmid or chromosome) of bont/B variants [20]. Multi-locus sequence typing (MLST) has been used to differentiate Group I serotype A strains
[21] and Group II serotype E strains [22]. These various genetic methods provide a baseline of understanding of phylogenetic relationships among Clostridia species. Improved DNA sequencing technologies and bioinformatic techniques now available allow researchers to compare strains at a higher level of resolution.

Botulinum neurotoxin-producing Clostridia have been identified from all continents except Antarctica. These microbes are often isolated from samples associated with human botulism cases (food poisonings or intestinal or wound infections), and researchers have also isolated BoNT-producing Clostridia from environmental samples including soils, honey, aquatic sediments and plants [17, 23-34]. The distribution of serotypes A-G can be obtained from publications based upon characterization of strains within different culture collections, environmental sampling and reported botulism cases. Identifying BoNT-producing strains from sources around the globe aids in understanding the frequency and geographic distribution of strains containing various toxin types.

The study represents a collaborative effort among researchers at many institutions to understand the diversity within BoNT-producing Clostridia. The study provides genomic sequence data and draft genome assemblies for strains (predominantly belonging to C. botulinum Groups I and II) representing diverse serotypes and geographic regions including isolates from botulism cases and environmental sources from Argentina, Australia, Canada, Finland, France, Greenland, Japan, Mauritius, Sweden and the US. These data are useful for determining neurotoxin gene cluster characteristics of BoNT-produding strains, the genomic backgrounds containing botulinum toxin genes, and the global distribution of strains expressing different toxin types. The study demonstrates that comparative genomic techniques differentiate BoNT-producing strains (including strains expressing the same toxin type or subtype) and illustrates the diversity of BoNTproducing strains (including the diversity of strains within Groups I and II). Knowledge of the diversity and phylogenetic relationships of BoNT-producing strains provides a framework for the study of these bacteria and can inform future research regarding topics such as the development of diagnostic tools and therapeutics.

\section{Methods}

\section{Genome sequencing, assembly and annotation}

Strains or purified DNA were kindly provided by numerous collaborators. Whole genome sequence data were generated with the Illumina sequencing technology (GAIIx). Genomes were assembled de novo via an in-house pipeline [35] that included adapter trimming with Trimmomatic [36], read error correction with BayesHammer [37] and contig assembly with SPAdes v3.0.0 [38]. Redundant contigs were removed with PSI-cd-hit [39], and short contigs 
( $<200 \mathrm{nt})$ were filtered out of assemblies. Illumina reads were mapped back to assemblies with BWA [40, 41], and single nucleotide polymorphisms were identified with GATK [42] and corrected if they passed a minimum depth and allele proportion. Assemblies were then improved with PILON [43] and IMAGE [44]. Assemblies were screened for obvious contamination by BLAST [45] searches against the NCBI non-redundant nucleotide database (contamination was removed from data for strain U21312). Relevant, published Clostridia species genomes were identified with the aid of the PATRIC database phylogeny viewer [46] and literature searches. Genome assemblies were downloaded from PATRIC [46] or GenBank [47] in fasta format (January 2015). The genome assembly of Acetobacter woodii DSM 1030 [GenBank:NC_016894] was downloaded for use as an outgroup. For inclusion in analyses, published genome assemblies needed to meet the following requirements: $<800$ contigs, presence of a near full-length 16S rRNA gene (>1300 nt) and presence of an $r p o B$ gene. All genome assemblies (newly sequenced and published strains) were annotated with Prokka [48] and evaluated with QUAST [49]. In addition to published genome assemblies, read files for ten Group I C. botulinum [50] genomes were included for the core genome sequence phylogenies of Group I strains.

\section{S rRNA gene phylogeny}

16S rRNA genes were parsed from Prokka output files. If multiple $16 \mathrm{~S}$ rRNA gene sequences were present in an assembly, the sequences from that assembly were clustered (99 \% identity) with USEARCH [51] and a representative sequence was chosen for that assembly. Representative sequences were aligned and masked with SSU-ALIGN [52]. Aligned and masked sequences were trimmed with mothur (filter.seqs command) [53] so the first and last position of each sequence included a base (not a gap character). A phylogeny was inferred with FastTree2 [54], an approximately-maximum-likelihood method, using the general time reversible model of nucleotide substitution and 1000 bootstrap replicates. The tree was viewed and rooted with the $A$. woodii DSM 1030 16S rRNA gene sequence in FigTree v1.4.2 [55].

\section{Phylogeny of concatenated marker genes}

Forty marker genes identified by specI (species identification tool) [56], a software package developed to delineate microbial species, were extracted from Prokka output files. Gene sequences of sufficient length (80\% of the length of $97.5 \%$ of the extracted sequences for each gene) were aligned with MUSCLE [57] and concatenated. The final alignment included gap characters for marker genes that could not be extracted from some genome assemblies. Alignment columns containing greater than $95 \%$ gap characters were filtered from the alignment with QIIME v1.6.0 [58]. Phylogenies were inferred on the concatenated alignments with FastTree2 as described above. The tree was viewed and rooted with A. woodii DSM 1030 in FigTree.

\section{Single nucleotide polymorphism (SNP) detection and phylogeny}

All genome assemblies were compared with the reference-independent single nucleotide polymorphism (SNP) approach, kSNP v2 [59]. kSNP was run with a kmer value of 21. SNPs identified in at least $50 \%$ of the analyzed genomes were used to infer a phylogeny. A phylogeny was inferred on the 40,582-character matrix with FastTree2 as described above. Referenceindependent SNP phylogenies were also generated for Group I and II strains. The Group I and II phylogenies were inferred upon core SNP matrices (Group I - 1,780character matrix, Group II - 35,382-character matrix) generated by kSNP, as described above.

SNP discovery was also performed by aligning assembled genomes to a reference assembly with NUCmer [60] and identifying SNPs from these alignments with NASP [61]. Illumina reads for ten Group I strains were aligned against the reference with BWA-MEM [41] and SNPs were called with the UnifiedGenotyper method in GATK [62]. SNP calls were filtered from the final matrix if the coverage at a position was less than $10 \times$ or if the proportion of reads matching the called SNP was less than 0.9. SNPs called from duplicated regions in the reference genome (identified by self alignments with NUCmer) were filtered from the SNP matrix. Phylogenies were inferred with RAxML v8.1.1 [63] using the general time reversible model of nucleotide substitution and the gamma distribution of rate heterogeneity. Ascertainment bias correction was applied to likelihood calculations [64] within RAxML. Bootstrap replicates were conducted using the rapid bootstrapping method in RAxML [65], and the number of bootstrap replicates was determined by using the RAxML extended majority-rule consensus tree criterion [66]. The tree was viewed and rooted in FigTree. C. botulinum strain Kyoto-F [GenBank:CP001581] was used as the reference genome for SNP detection for Group I C. botulinum/C. sporogenes resulting in a 200,641-character core genome SNP matrix called from a 1,708,420-character core genome alignment (positions that passed quality filtering). The core genome phylogeny for Group I was rooted with the clade including $C$. sporogenes and $C$. botulinum serotype $B$ strains based upon the concatenated marker genes and kSNP phylogenies of all genomes included in this study as well as reference-based SNP phylogenies including C. tetani strains as an outgroup (data not shown). C. botulinum strain Eklund 17B [GenBank:CP001056, CP001057] was used as the reference genome for SNP 
detection for Group II C. botulinum resulting in a 197,688-character SNP matrix called from a 2,609,405character core genome alignment. The core genome phylogeny for Group II was rooted with the clade containing strains Eklund 202F, KAPB-3, Eklund 17B and CDC 66177 based upon the concatenated marker genes and kSNP phylogenies of all the genomes included in this study as well as reference-based SNP phylogenies including C. saccharobutylicum DSM 13864 [GenBank:CP006721] as an outgroup (data not shown). Reference-based SNP detection and core genome phylogenies with alternate reference genomes were also generated: Group I - C. sporogenes ATCC 15579 [GenBank:ABKW00000000], Group II - C. botulinum strain Alaska E43 [GenBank:CP001078].

\section{Analysis of phylogenies}

The consistency index and retention index for core genome phylogenies was computed with the $\mathrm{R}$ [67] package phangorn [68]. Compare2Trees [69] was used to compare tree topologies for Groups I and II core genome phylogenies and for $16 \mathrm{~S}$ rRNA gene, concatenated marker genes and kSNP phylogenies. The overall topological score is reported as a measure of tree topology similarity.

\section{SNP and homoplasy density in C. botulinum Groups I and II} SNP density and homoplasy density ratio were computed using the SNP matrices and core genome phylogenies produced by NASP and RAxML (see above) to provide insight into recombination within Groups I and II. SNP density was determined by counting the number of parsimony informative SNPs present in $1 \mathrm{~kb}$ nonoverlapping segments of the core genome for each Group. The homoplasy density ratio was computed by dividing the number of parsimony informative SNPs with a retention index below 0.5 (calculated with PAUP* 4.0 beta [70]) by the total number of parsimony informative SNPs in $1 \mathrm{~kb}$ segments of the core genome for each Group. The SNP density and homoplasy density ratio values across the reference genomes were plotted with Circos [71]. Histograms of the homoplasy density ratio values of $1 \mathrm{~kb}$ segments of the core genome (only $1 \mathrm{~kb}$ segments with at least ten parsimony informative SNPs are included in the histogram) are presented for Groups I and II.

\section{Pairwise genomic comparisons - average nucleotide identity}

The average nucleotide identity between pairs of genome assemblies (analysis included chromosomal and extrachromosomal sequences) was computed with JSpecies [72] using the MUMmer calculation (ANIm) and default settings. A histogram of ANIm values of inter- and intra-Group comparisons of C. botulinum Groups I, II,
III and VI as well as C. perfringens and C. tetani was created with matplotlib [73]. These Groups were chosen for comparison because they contain strains that produce botulinum neurotoxins or the tetanus toxin and/or have multiple sequenced genomes within the Group/species.

\section{Gene content analyses with LS-BSR}

Genome assemblies were processed with the largescale BLAST score ratio pipeline (LS-BSR) [74] using the BLAT [75] alignment option and default parameters to assess the gene content of Group I and II strains. Group I and II genomes were clustered based on BSR values using an average linkage algorithm implemented in the MultiExperiment Viewer $(\mathrm{MeV})$ [76]. Dendrograms produced by $\mathrm{MeV}$ were viewed and rooted in Figtree. Additionally, cold shock protein encoding genes were screened against Group II genomes using the LS-BSR approach. Cold shock protein encoding genes were downloaded from PATRIC [46] for $C$. botulinum strains ATCC 3502 (PATRIC IDs fig|413999.7.peg.282, fig|413999.7.peg.1366, fig|413999.7.peg.1745), Eklund 17B (fig|508765.6.peg.1446) and Eklund (fig|445337.5.peg.496, fig|445337.5.peg.1216) as well as $C$. beijerinckii strain NCIMB 8052 (fig|290402.41.peg.2890, fig|290402.41.peg.3037), and C. butyricum strains 5521 (fig|447214.4.peg.3792) and BL5262 (fig|632245.3.peg.2731).

\section{Phylogeny of concatenated multi-locus sequence typing (MLST) genes}

Genes for multilocus-sequence typing (MLST) were selected from previous MSLT studies. The MLST profile for Group I strains ( $a c e K$, $a r o E, h s p, m d h, o p p B$, rec $A$ and $r p o B$ ) was selected from Jacobson and colleagues [21]. The MLST profile for Group II strains (16S rRNA gene, atpD, guaA, gyrB, ilvD, lepA, opp $B$, $p t a, p y c, r e c A, r p o B, \operatorname{trp} B$ and tuf) was adapted from MacDonald and colleagues [22], though 23S rRNA gene sequences were not included in the analysis. These gene sequences were downloaded from PubMLST C. botulinum database $[77,78]$ or GenBank. MLST genes were extracted from genome assemblies with BLAST searches. Gene sequences were aligned with MUSCLE and concatenated for phylogenetic reconstruction. Phylogenies were inferred with FastTree2 as described above (see 16S rRNA gene phylogeny methods). Two genome assemblies included in the Group I analyses were missing one gene from the MLST profile - C. botulinum CDC 54091 had no recA gene and $C$. botulinum Af84 had no $m d h$ gene. Gap characters were inserted into the gene alignments for these two genomes. 


\section{bont gene cluster analyses}

Botulinum neurotoxin gene sequences were extracted from Prokka output files. Previously published bont gene and tetanus toxin gene sequences downloaded from GenBank were also included in the analyses. Sequences longer than 3500 nucleotides were aligned with MUSCLE and trimmed with Mothur (filter.seqs command) so the first and last position of each sequence included a base (not a gap character). A phylogeny was constructed with Fasttree 2 as described above (see 16S rRNA gene phylogeny methods). The tree was viewed and rooted with the tetanus toxin gene clade in FigTree. Annotated genome assemblies were investigated to determine the putative bont gene cluster type $\left(h a+\right.$ or orf $\left.X_{+}\right)$ and location within the genome. Newly sequenced genomes were aligned to previously published genome assemblies with progressiveMauve [79] to aid in understanding putative bont gene cluster locations. The presence of a plasmid-specific marker gene $(P L-6)$ in all genome assemblies was determined with BLAST searches (hits above $80 \%$ identity) of assembled genomes against a putative DNA primase gene [GenBank:CP000940.1, locus CLD_A0039] [80].

\section{Results}

A total of 59 new draft genome assemblies were generated for strains isolated from six different continents (Table 1 and (Additional file 1: Table S1)). The strains were previously isolated from botulism cases and environmental samples and include 32 bont/A, six bont/B, nine bont/E, one bont/F, five bont/A1(B), two bivalent bont/A2f4, one bivalent bont/Bf and three strains that did not contain botulinum neurotoxin genes in the draft genome assemblies (two strains within Group I and one strain not within the six BoNT-producing Groups). Information regarding previously published genomes included in this study is presented (Additional file 2: Table S2). Fig. 1 illustrates the countries of origin of BoNT-producing strains sequenced and/or analyzed in this study and demonstrates the small number of whole genome sequences available for strains originating from Asia and Africa. This study is one of the most comprehensive comparative genomic analyses of C. botulinum and closely-related strains performed to date.

\section{Delineation of Clostridia species/Groups}

The phylogenetic relationships and diversity of BoNTproducing strains were evaluated with phylogenies of the 16S rRNA gene, concatenated marker genes, and SNPs and with average nucleotide identity. A phylogeny of $16 \mathrm{~S}$ rRNA gene sequences extracted from each genome assembly indicates that the newly sequenced strains (except for non-BoNT-producing strain U20725 that groups near the Group IV strain in Fig. 2) are closely related to strains within C. botulinum Groups I and II (Fig. 2). Group I includes serotypes A, B and F as well as bivalent and non-toxic strains. Group II includes serotypes B, E and F. BoNT-producing strains are colorcoded by serotype in Fig. 2, which illustrates evidence of horizontal gene transfer and that non-BoNT-producing strains are closely related to BoNT-producing strains (e.g. C. sporogenes in Group I and C. novyi in Group III).

The deeper relationships of BoNT-producing Clostridia strains were also evaluated with phylogenies inferred on a concatenation of 40 marker genes identified by Mende and colleagues [56] for delineation of species (i.e. universal MLST-like approach for identifying microbial species) and a SNP-matrix produced by kSNP (Fig. 3). The 16S rRNA gene, concatenated marker genes and kSNP phylogenies show similar overall topologies (overall topological scores computed by Compare2Trees range from 66 to $78 \%$ ). All phylogenies indicate that Group III C. botulinum strains are an outgroup to all other BoNT-producing strains and that a close relationship exists between C. tetani strains and C. botulinum Group I strains. The concatenated marker genes and kSNP phylogenies provide greater resolution than the $16 \mathrm{~S}$ rRNA gene phylogeny and indicate that multiple clades are present in C. botulinum Groups I, II and III, which is consistent with previous findings [11, 21, 22, 50, 81-84]. Among the biggest differences between the trees is the inclusion of the Group IV C. argentinense strain in a clade with the Group I strains in the $16 \mathrm{~S}$ rRNA gene and kSNP trees, while the same Group IV $C$. argentinense strain falls into a clade with Groups II, V, VI in the concatenated marker genes phylogeny.

Average nucleotide identity (MUMmer method ANIm), a method that can be applied to delineate species [72], was calculated to determine diversity at the genomic level within and between BoNT-producing Groups (Fig. 4 and (Additional file 3: Table S3)). Richter and Rossello-Mora [72] suggested that ANIm values above $95-96 \%$ may be applied to define species, though the authors noted some exceptions, and ANIm values between 93 and $96 \%$ may fall into an intermediate zone of species classification [85]. Minimum ANIm values in Groups I, II and III fall below the threshold of 95-96 \%, and minimum ANIm values for Group I and Group III fall below $93 \%$, which is indicative of the relatively high diversity within these Groups [11, 83, 84]. For comparison, the minimum ANIm values for strains within $C$. perfingens and C. tetani (97.02 and 99.13, respectively) are well above the suggested species cutoff value, and looking outside of the Clostridia, Escherichia coli O157:H7 str. EC869 and E. fergusonii ATCC 35469 share 92.56 ANIm (data not shown). Multiple clades are present in C. botulinum Groups I and II in the concatenated marker genes and kSNP phylogenies 
Table 1 Information regarding newly sequenced strains

\begin{tabular}{|c|c|c|c|c|c|c|c|c|c|c|}
\hline Genome & Accession \# & Group & BoNT & Strain/alternate ID & $\begin{array}{l}\text { BoNT cluster } \\
\text { type }\end{array}$ & $\begin{array}{l}\text { BoNT cluster } \\
\text { locationa }^{a}\end{array}$ & Genomic site $^{a}$ & Year & Origin & Location \\
\hline C. botulinum 20386 & LFRD00000000 & I & $\mathrm{A} 1$ & VPI 7124 & hat & chr & oppA/BrnQ & & soil & USA:Virginia \\
\hline C. botulinum 20389 & LFOO00000000 & । & $\mathrm{A} 1$ & ATCC 449 & hat & chr & oppA/BrnQ & & & \\
\hline C. botulinum 20412 & LFOT00000000 & । & $\mathrm{A} 1$ & KF Meyer 126 & hat & chr & oppA/BrnQ & 1921 & spinach (FB) & USA:Indiana \\
\hline C. botulinum 20414 & LFOU00000000 & । & $\mathrm{A} 1$ & Prevot 910 & hat & chr & oppA/BrnQ & 1953 & bovine botulism & France \\
\hline C. botulinum 20424 & LFOV00000000 & I & $\mathrm{A} 1$ & Prevot Dewping & hat & chr & oppA/BrnQ & & & \\
\hline C. botulinum 20427 & LFOW00000000 & । & $\mathrm{A} 1$ & Prevot 697B & hat & chr & oppA/BrnQ & 1952 & cat gut & Sweden \\
\hline C. botulinum 20503 & LFOY00000000 & । & $\mathrm{A} 1$ & McClung 844 & hat & chr & oppA/BrnQ & $<1930$ & & \\
\hline C. botulinum 20504 & LFOZ00000000 & । & $\mathrm{A} 1$ & KF Meyer 33 & hat & chr & oppA/BrnQ & 1920 & ripe olives & USA:Tennessee \\
\hline C. botulinum AM1295 & LFPI00000000 & 1 & $\mathrm{~A} 1$ & & hat & chr & oppA/BrnQ & & suspected reference strain & \\
\hline C. botulinum U21312 & LFQF00000000 & । & $\mathrm{A} 1$ & SU0729 & orf $X+$ & chr & $\operatorname{ars} C$ & 1987 & soil & Argentina \\
\hline C. botulinum 10148 & LFOK00000000 & । & $\mathrm{A} 1(\mathrm{~B})$ & CDC 1744 & A1-orfX+, (B)-ha+ & chr & A1-arsC, (B)-oppB/BrnQ & 1977 & $\mathrm{IB}$ & USA:Pennsylvania \\
\hline C. botulinum 20391 & LFOP00000000 & 1 & $\mathrm{~A} 1(\mathrm{~B})$ & Hall 183 & A1-orfX+, (B)-ha+ & chr & A1-arsC, (B)-oppB/BrnQ & 1922 & corn (FB) & USA:Colorado \\
\hline C. botulinum 20396 & LFOQ00000000 & । & $\mathrm{A} 1(\mathrm{~B})$ & Hall 4834 & A1-orf $X+,(B)-h a+$ & chr & A1-arsC, (B)-oppB/BrnQ & 1931 & spinach (FB) & USA:Nebraska \\
\hline C. botulinum 20397 & LFOR00000000 & । & $\mathrm{A} 1(\mathrm{~B})$ & Hall 8388A & A1-orf $X+$, (B)-ha+ & chr & A1-arsC, (B)-oppB/BrnQ & 1935 & chili pepper (FB) & USA:New Mexico \\
\hline C. botulinum 20398 & LFOS00000000 & । & $\mathrm{A} 1(\mathrm{~B})$ & Hall 8857Ab & A1-orfX+, (B)-ha+ & chr & A1-arsC, (B)-oppB/BrnQ & 1935 & corn (FB) & USA:Nebraska \\
\hline C. botulinum Mauritius & LFPL00000000 & । & $A 2$ & Mauritius & orf $x+$ & chr & $\operatorname{arsC}$ & & fish (FB) & Mauritius \\
\hline C. botulinum U21063 & LFPN00000000 & । & $\mathrm{A} 2$ & SU1937 & orf ++ & chr & $\operatorname{arsC}$ & 2009 & soil & Argentina \\
\hline C. botulinum U21067 & LFPQ00000000 & 1 & $A 2$ & SU1274 & orf ++ & chr & $\operatorname{ars} C$ & 1997 & soil & Argentina \\
\hline C. botulinum U21068 & LFPR00000000 & । & $A 2$ & SU1917 & orf $x+$ & chr & $\operatorname{arsC}$ & 2009 & soil & Argentina \\
\hline C. botulinum U21069 & LFPS00000000 & । & $A 2$ & SU1887 & orf $X+$ & chr & $\operatorname{arsC}$ & 2007 & soil & Argentina \\
\hline C. botulinum U21070 & LFPT00000000 & 1 & $\mathrm{~A} 2$ & SU1275 & orfX+ & chr & $\operatorname{ars} C$ & 1997 & soil & Argentina \\
\hline C. botulinum U21075 & LFPU00000000 & । & $A 2$ & SU1934 & orf $X+$ & chr & $\operatorname{ars} C$ & 2010 & soil & Argentina \\
\hline C. botulinum U21077 & LFQY00000000 & । & $A 2$ & SU1259 & orf $X+$ & chr & $\operatorname{arsC}$ & 1996 & soil & Argentina \\
\hline C. botulinum U21078 & LFQZ00000000 & 1 & $\mathrm{~A} 2$ & SU1891 & orfX+ & chr & $\operatorname{ars} C$ & 2007 & soil & Argentina \\
\hline C. botulinum U21082 & LFPW00000000 & । & $A 2$ & SU1054 & orf $X+$ & chr & $\operatorname{arsC}$ & 1998 & soil & Argentina \\
\hline C. botulinum U21084 & LFPX00000000 & । & $\mathrm{A} 2$ & SU1072 & orf $X+$ & chr & $\operatorname{arsC}$ & 1998 & soil & Argentina \\
\hline C. botulinum U21086 & LFPY00000000 & । & A2 & SU1064 & orf $X+$ & chr & $\operatorname{arsC}$ & 1998 & soil & Argentina \\
\hline C. botulinum U21088 & LFPZ00000000 & I & $\mathrm{A} 2$ & SU1074 & orf ++ & chr & $\operatorname{arsC}$ & 1998 & soil & Argentina \\
\hline C. botulinum U21089 & LFQA00000000 & । & A2 & SU1112 & orf $X+$ & chr & $\operatorname{arsC}$ & 1995 & soil & Argentina \\
\hline C. botulinum U21306 & LFRC00000000 & । & A2 & SU0801 & orf $x+$ & chr & $\operatorname{arsC}$ & 2010 & soil & Argentina \\
\hline C. botulinum U21307 & LFQB00000000 & 1 & $A 2$ & SU0998 & orf $X+$ & chr & $\operatorname{ars} C$ & 1994 & soil & Argentina \\
\hline
\end{tabular}


Table 1 Information regarding newly sequenced strains (Continued)

\begin{tabular}{|c|c|c|c|c|c|c|c|c|c|c|}
\hline C. botulinum U21309 & LFQC00000000 & 1 & $\mathrm{~A} 2$ & SU0807 & orf $X+$ & chr & arsC & 1987 & soil & Argentina \\
\hline C. botulinum U21310 & LFQD00000000 & 1 & $A 2$ & SU0994 & orfX+ & chr & $\operatorname{ars} \mathrm{C}$ & 1994 & soil & Argentina \\
\hline C. botulinum U21311 & LFQE00000000 & I & $A 2$ & SU0634 & orfX+ & $\mathrm{chr}$ & $\operatorname{ars} C$ & 1980 & soil & Argentina \\
\hline C. botulinum U21729 & LFQG00000000 & I & $A 2$ & SU0635W & orf $X+$ & chr & arsC & & soil & Argentina \\
\hline C. botulinum U21064 & LFPO00000000 & I & $\mathrm{A} 2 \mathrm{f} 4$ & SU1306 & A2-orfX+, F4-orfX+ & $\mathrm{chr}$ & A2-arsC, F4-PulE & 1998 & soil & Argentina \\
\hline C. botulinum U21076 & LFPV00000000 & I & $\mathrm{A} 2 \mathrm{f} 4$ & SU1304 & A2-orfX+, F4-orfX+ & $\mathrm{chr}$ & A2-arsC, F4-PulE & 1998 & soil & Argentina \\
\hline C. botulinum U21087 & LFRA00000000 & I & A3 & SU1169 & orf ++ & & & 1998 & soil & Argentina \\
\hline C. botulinum U21181 & LFRB00000000 & 1 & A3 & SU0945 & orfX+ & & & 1992 & soil & Argentina \\
\hline C. botulinum 20497 & LFOX00000000 & 1 & B1 & Hall 80 & hat & chr & oppA/BrnQ & 1918 & beans (FB) & USA:Illinois \\
\hline C. botulinum 10159 & LFOL00000000 & । & B2 & ATCC 17843 (B5) & hat & chr & oppA/brnQ & & & \\
\hline C. botulinum 20506 & LFQV00000000 & 1 & B3 & CDC 795 & hat & chr & oppA/BrnQ & & & USA:Michigan \\
\hline C. botulinum 10258 & LFON00000000 & 1 & $B 5 f 2$ & An436 & B5-ha+, F2-orfX+ & plasmid & & & $\mathrm{IB}$ & Sweden \\
\hline C. botulinum AM1195 & LFPH00000000 & । & B6 & AM1195 & hat & plasmid & & 1987 & $\mathrm{IB}$ & Australia \\
\hline C. botulinum AM370 & LFPJ00000000 & 1 & B6 & AM370 & hat & plasmid & & 1979 & salted fish & Australia \\
\hline C. botulinum AM553 & LFPK00000000 & 1 & B6 & AM553 & hat & plasmid & & 1981 & & Australia \\
\hline C. botulinum 20552 & LFPF00000000 & I & F1 & Walls $8 \mathrm{G}$ & orfX+ & chr & arsC & 1968 & crabs (ENV) & USA:Virginia \\
\hline C. sp. U21066 & LFPP00000000 & 1 & NT & SU1575NT & & & & 1999 & soil - colony variant of SU1575 & Argentina \\
\hline C. sporogenes U20719 & LFPM00000000 & I & NT & ATCC 19404 & & & & & & \\
\hline C. botulinum 20536 & LFPA00000000 & $\|$ & E1 & CDC KA-95B & orfX+ & chr & $\operatorname{rar} \mathrm{A}$ & & & \\
\hline C. botulinum 20541 & LFPB00000000 & $\|$ & E1 & L-572 & orfX+ & chr & $\operatorname{rarA}$ & & forest soil & USA:Washington \\
\hline C. botulinum 20547 & LFPD00000000 & $\|$ & E1 & Prevot Ped 1 & orfX+ & chr & $\operatorname{rarA}$ & & sea mud & Greenland \\
\hline C. botulinum 20675 & LFPG00000000 & $\|$ & E1 & ATCC 9564 & orfX+ & chr & $\operatorname{rar} \mathrm{A}$ & 1961 & smoked salmon & Canada \\
\hline C. botulinum K15 & LFQW00000000 & $\|$ & E1 & K15 & orfX+ & chr & $\operatorname{rarA}$ & 1995 & trout & Finland \\
\hline C. botulinum 20544 & LFPC00000000 & $\|$ & E2 & CDC 5247 & orfX+ & chr & rarA & & & USA:Alaska \\
\hline C. botulinum 10178 & LFOM00000000 & $\|$ & E3 & 211 & orf $X+$ & chr & $\operatorname{rar} \mathrm{A}$ & & lake sediment & Japan \\
\hline C. botulinum 20549 & LFPE00000000 & $\|$ & E3 & Prevot R81-3A & orfX+ & chr & $\operatorname{rar} \mathrm{A}$ & & & France \\
\hline C. botulinum K3 & LFQX00000000 & $\|$ & E3 & K3 & orfX+ & chr & $\operatorname{rarA}$ & 1995 & trout & Finland \\
\hline C. sp. U20725 & LFRG00000000 & & NT & ATCC 25772 & & & & & & \\
\hline
\end{tabular}

$F B$ foodborne isolate, $I B$ infant botulism case, $E N V$ environmental isolate

a putative information based upon draft genome assemblies 


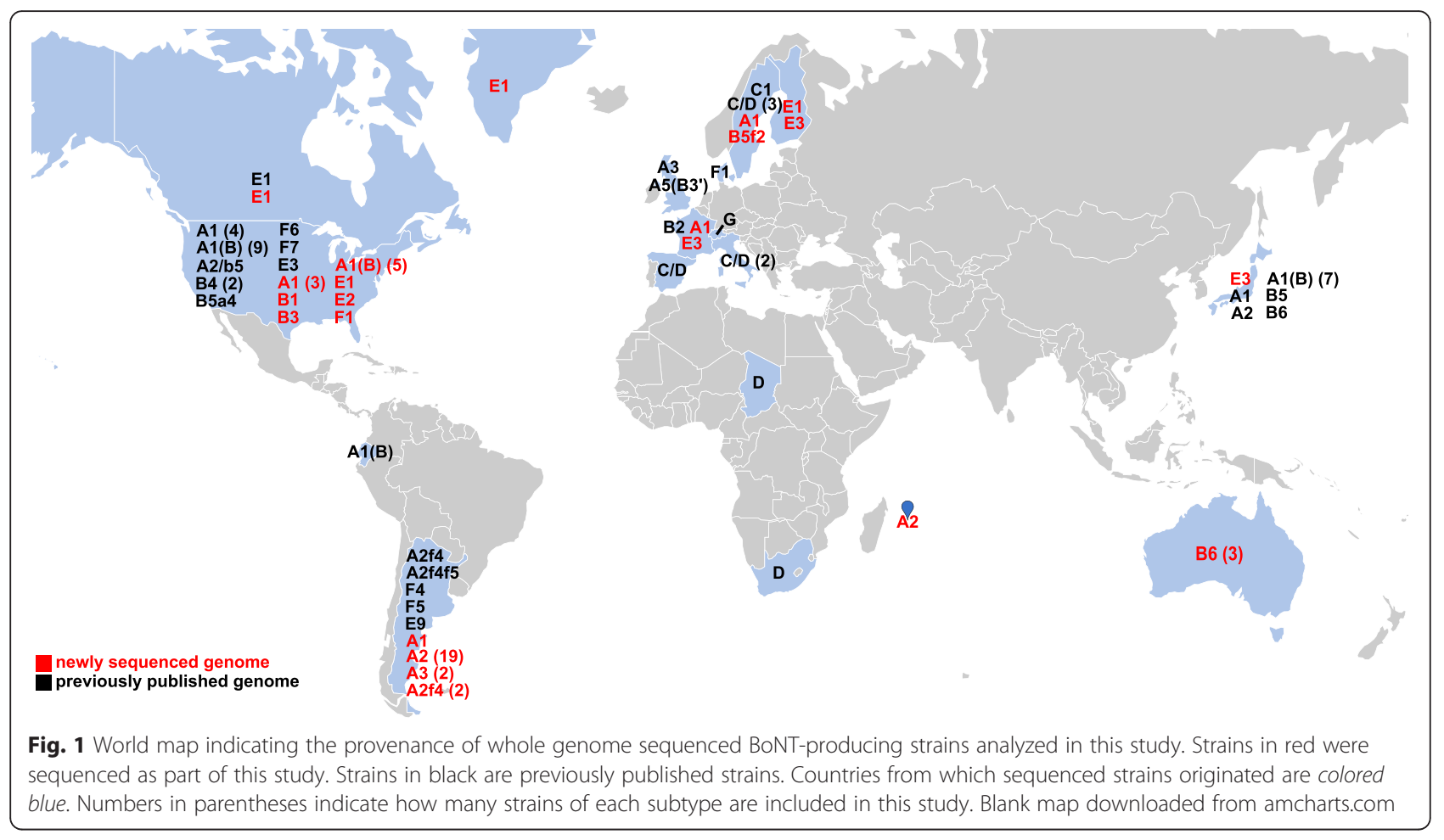

(Fig. 3) as well as in core genome phylogenies (discussed below). While minimum ANIm values fall below the species delineation value when all genomes in either Group I or Group II are considered, ANIm values are above $95 \%$ within each of the two distinct clades present in Group I, and ANIm values are above $97 \%$ within each of the two distinct clades present in Group II. By this measure, both Groups I and II could be considered to encompass multiple species, subspecies or genomovars (distinct groups on the genomic level but similar phenotypically) [86]. Regardless of the assigned nomenclature, the genomic diversity within the Groups is evident.

\section{Phylogeny of Group I C. botulinum/C. sporogenes}

Many of the newly sequenced strains belong to the Group I C. botulinum/C. sporogenes. To provide a highresolution investigation of the relationships of 93 Group I strains, a maximum likelihood phylogeny was estimated from an alignment of $\sim 200,000$ core genome SNPs (Fig. 5). Core genome phylogenies generated with an alternative reference genome as well as kSNP are also presented (Additional file 4: Figure S1). Group I includes diverse BoNT/A,/B and/F-producing strains as well as non-BoNT-producing strains that fall into multiple clades, which is consistent with previous studies $[1,50$, 82, 84]. When considering ANIm values, strains within the $C$. sporogenes-BoNT/B-producing outgroup (bottom of Fig. 5) share ANIm values above $95 \%$. The strains in the remainder of the tree also share ANIm values above $95 \%$. However, when comparing all Group I strains ANIm values fall below $95 \%$ (minimum of 92.2\%) (as mentioned above), indicating the high genomic diversity present in Group I. The core genome phylogeny provides a framework for investigating Group I strains including the variation in genomic backgrounds expressing the same toxin type or subtype.

BoNT/A-producing strains (subtypes A1, A2 and A3) belong to multiple clades of the Group I core genome phylogeny presented in Fig. 5. Subtype A1 strains show considerable genomic diversity. The newly sequenced orf $X+$ bont/A1 strain U21312 from Argentina belongs to a clade that includes the orf $X+$ bont/A1 strain CDC 297 (two assemblies for this strain from New York, labeled CDC 297 and CFSAN002368, were included to compare results when analyzing the same strain sequenced and assembled by different groups and methods - the strains group together in the phylogeny) but is less closely related to strain CDC 297 than other bont/A2 and bivalent strains. Two clades of bont/A1(B) strains (orf $X+$ bont A1) are found in the Group I phylogeny. Five bont/ A1(B) strains (10148, 20397, 20398, 20396 and 2039) from the United States are closely related to other bont/ A1(B) strains from Ecuador, Japan and the US while three recently published bont/A1(B) strains isolated from infant botulism cases in Japan [50] fall into a distinct clade. The ha+ bont/A1 strains included in this study belong to one clade that also includes a bont/B1 strain, a 


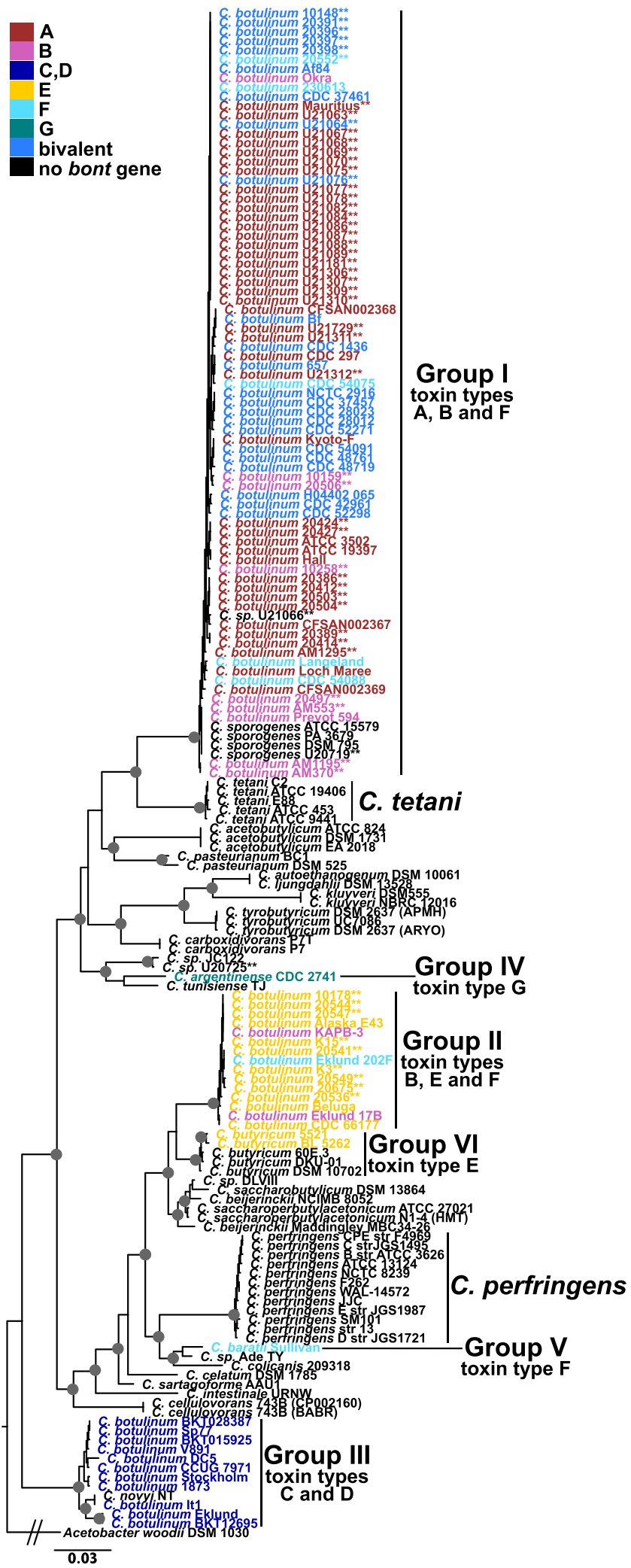

Fig. 2 (See legend on next page.) 
(See figure on previous page.)

Fig. 2165 rRNA gene phylogeny of Clostridia strains. A phylogeny inferred with FastTree2 [54] on near-full-length 16S rRNA gene sequences extracted from genome assemblies and aligned and masked with ssu-align [52]. The tree is rooted with Acetobacter woodii DSM 1030 [GenBank:NC_016894]. Strains are color-coded by botulinum neurotoxin serotype. Stars indicate newly sequenced (this study) strains. Groups I-VI, Clostridium perfringens and C. tetani clades are labeled. Gray circles indicate bootstrap values over $90 \%$

bont/F4 strain and a strain that does not contain a botulinum neurotoxin gene (strain U21066 is a colony variant of a bont/A strain). Three ha+ bont/A1 subclades include previously published genomes, but strain 20412 isolated from spinach associated with a food botulism case in the US (Indiana) is less closely related to these previously sequenced $h a+b o n t / \mathrm{A} 1$ strains.

Argentinian subtype bont/A2 strains analyzed in this study were all isolated from soils. Many of the newly sequenced bont/A2 strains are closely related to the bont/ A2 strain C. botulinum Kyoto-F (Fig. 5). However, the newly sequenced bont/A2 Argentinian strains U21069, U21089, U21063 and U21075 form a distinct but closely related clade to the bont/A2 group that includes the Kyoto-F strain. Additionally, two newly sequenced Argentinian bont/A2 strains (U21311 and U21729) are distantly related to the previously mentioned bont/A2 strains, but appear to be more closely related to $\operatorname{orf} X+$ bont/A1 and bivalent strains. One newly sequenced bont/A2 strain from the Republic of Mauritius, an island country off the eastern coast of Africa, is not closely related to any of the Argentinian bont/A2 strains or the Kyoto-F strain. The diversity of the genomic backgrounds in which bont/A2 genes are found is evident from this analysis.

Prior to this study, only one genome assembly for a strain producing BoNT/A3, C. botulinum strain Loch Maree isolated in Scotland [87, 88], had been published. The Loch Maree strain is not closely related to any other whole genome sequenced strain (Fig. 5). Two Argentinian bont/A3 strains (U21087 and U21181) sequenced as part of this study are distantly related to the Loch Maree strain and are most closely related to bivalent bont/Af strains from Argentina.

BoNT/B-producing strains are also found in multiple clades in the Group I phylogeny. The BoNT/B1-producing strain 20497 is not closely related to the published whole-genome-sequenced BoNT/B1-producing strain, $C$. botulinum Okra, but instead falls within a clade that includes $h a+$ bont/A1 strains. Newly sequenced BoNT/B2producing strain 10159 and BoNT/B3-producing strain 20506 are not closely related to other BoNT/B-producing strains. The distantly related clade at the bottom of the Group I core genome phylogeny includes C. sporogenes strains and $C$. botulinum strains that produce BoNT/B2 and/B6 subtypes. The newly sequenced strains include three BoNT/B6-producing strains from Australia that are closely related to the Japanese BoNT/B6- producing strain Okayama 2011 (and Japanese bont/B6 strain Osaka05 not included in this study) [50]. Interestingly, the acquisition or loss of the ability to produce BoNT/B within the C. sporogenes-bont/B clade appears to be plasmid-mediated, which is consistent with findings of Weigand and colleagues [84]; although, evaluation of additional strains would be needed to confirm this observation.

The bont/F1 strain 20552 (from the United States) is closely related to previously published bont/F1 strains F230613 and Langeland (from Denmark). Strain 20552 was isolated from an environmental source (crabs) while the Langeland strain was isolated from duck liver paste associated with a food botulism case. The bont/F4 strain CDC 54088 and bont/F5 strain CDC 54075 (both from Argentina) are found in different clades and are not closely related to the serotype F1 strains.

Bivalent strains are found within two clades in the Group I phylogeny. A clade of closely related bivalent strains is nested within the clade just above the basal clade at the bottom of Fig. 5. This bivalent clade includes two strains that produce both BoNT/A and/B (strains CDC 657 and CDC 1436) as well as the BoNT/ B5f2-producing strains 10258 (from Sweden) and Bf. The second bivalent clade includes bont/A2f4 strains U21064 and U21076 from Argentina that are closely related to previously published bivalent bont/A2f4 and trivalent bont/A2f4f5 strains also from Argentina.

The core genome phylogeny illustrates the diversity of whole genome sequenced Group I strains. MLST is a comparative method available to many laboratories that does not require whole genome sequencing. To allow for the comparison of strains included in this study with published MLST data, a phylogeny of concatenated MLST genes including 83 sequence types (ST) available through the PubMLST C. botulinum database $[77,78]$ is included (Additional file 5: Figure S2). Similar to the core genome phylogeny (Fig. 5), the concatenated MLST gene phylogeny separates the Group I strains into two main clades. Sequence types associated with BoNT-producing and non-BoNT-producing strains fall into both main clades. C. sporogenes and BoNT/B-producing strains and two sequence types associated with serotype A strains (ST5 and ST17) fall into the clade corresponding to the C. sporogenes and bont/B strain clade in the core genome phylogeny (Fig. 5). Genome assemblies were not available for the two serotype A strains in this clade that might assist in further understanding the relationships of these strains. 

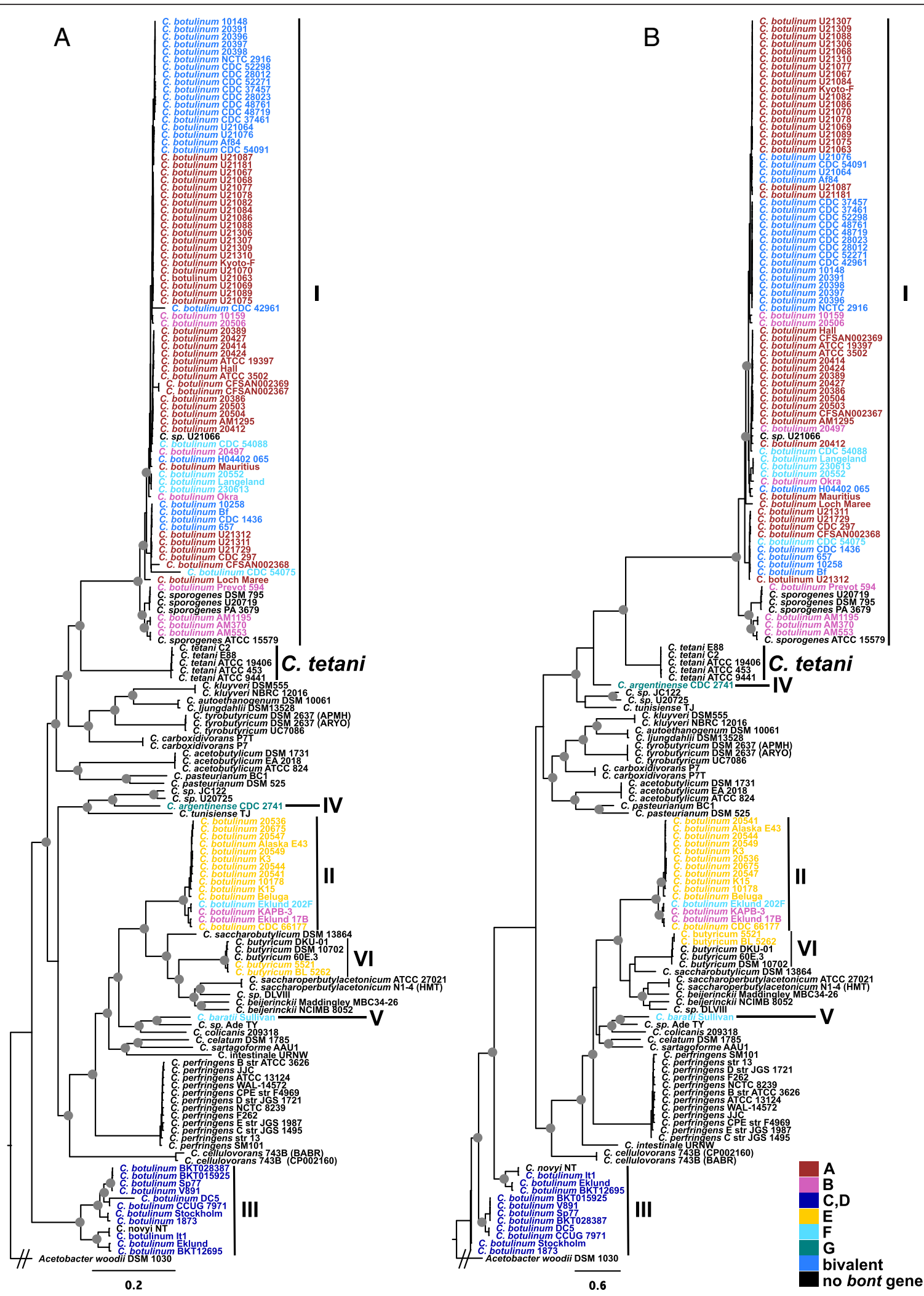

Fig. 3 (See legend on next page.) 
(See figure on previous page.)

Fig. 3 Phylogenies of Clostridia strains inferred on (a) concatenated marker genes and (b) a SNP matrix. Phylogenies inferred with FastTree2 [54] on (a) 40 concatenated marker genes for the delineation of species (specl) [56] aligned with MUSCLE [57] and (b) a SNP-matrix including SNPS identified in at least $50 \%$ of the analyzed strains produced by kSNP v2 [59]. The trees are rooted with Acetobacter woodii DSM 1030 [GenBank:NC_016894]. Strains are color-coded by botulinum neurotoxin serotype. Groups I-VI, Clostridium perfringens and C. tetani clades are labeled. Gray circles indicate bootstrap values over $90 \%$

\section{Phylogeny of Group II C. botulinum}

Newly sequenced strains also included BoNT/E-producing isolates belonging to the Group II C. botulinum. The relationships and diversity of 15 Group II strains were investigated with a maximum likelihood phylogeny inferred from an alignment of $\sim 200,000$ core genome SNPs (Fig. 6). Phylogenies generated with an alternate reference genome as well as kSNP are also presented (Additional file 6: Figure S3). Two distinct clades are apparent in the Group II phylogeny. ANIm values are above $97 \%$ within each major clade but fall below the species-delineating threshold of $95 \%$ (minimum of $\sim 93.8 \%$ ) when all Group II strains are considered, which illustrates the genomic diversity within the Group. The two major clades include a clade of only BoNT/E-producing strains and a clade of BoNT/B,/E and/F-producing strains. The newly sequenced serotype E strains fall into a clade comprised entirely of BoNT/
E-producing strains representing Canada, Finland, France, Greenland, Japan and the US. The second major clade includes BoNT/B4,/E9 and/F6-producing strains. The BoNT/E9-producing strain CDC 66177 from Argentina falls into the clade with BoNT/B and/ F-producing strains from the US, which is consistent with microarray hybridization profile analysis [83] and SNP analysis [89].

To allow for comparison of strains included in this study with published MLST data for other strains, a phylogeny of concatenated MLST genes including 41 C. botulinum serotype E strains presented in MacDonald et al. [22] is included (Additional file 7: Figure S4). Similar to the core genome phylogeny, the MLST phylogeny separates the Group II strains into two main clades: one that includes only bont/E strains and one that includes bont/B and/F strains as well as the bont/E9 CDC6617 strain.
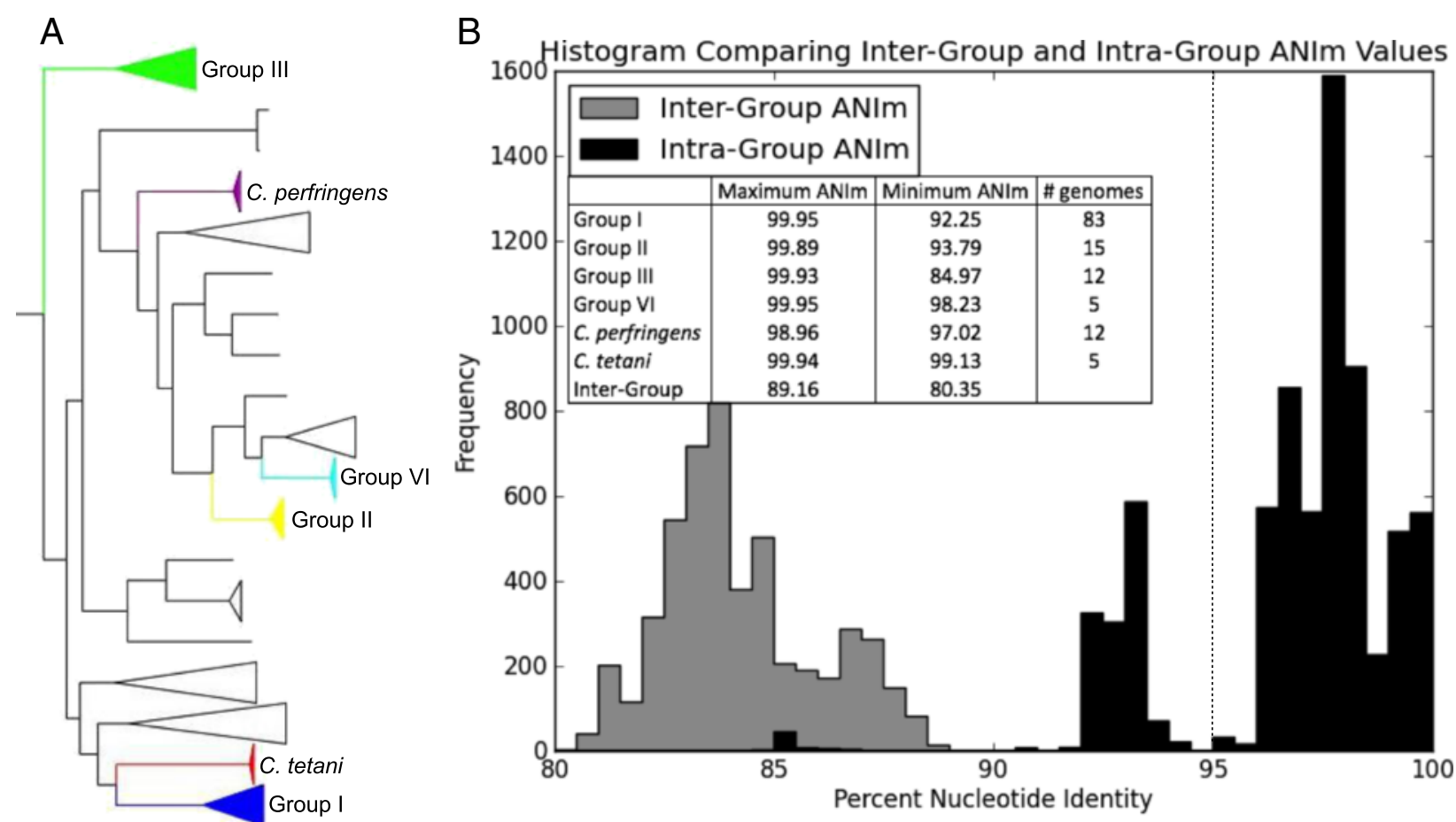

Fig. 4 Pairwise genomic comparisons with average nucleotide identity. a A dendrogram highlighting clades of Clostridia compared by the average nucleotide identity (MUMmer calculation) method (ANIm) with Jspecies [72] and included in the histogram presented in (b). b A histogram of inter-Group and intra-Group ANIm values. The vertical dotted line at $95 \%$ nucleotide identity indicates the cutoff for species delineation suggested by Richter and Rosello-Mora [72]. The inset table displays maximum and minimum percent nucleotide identity within each analyzed clade and between all analyzed clades 


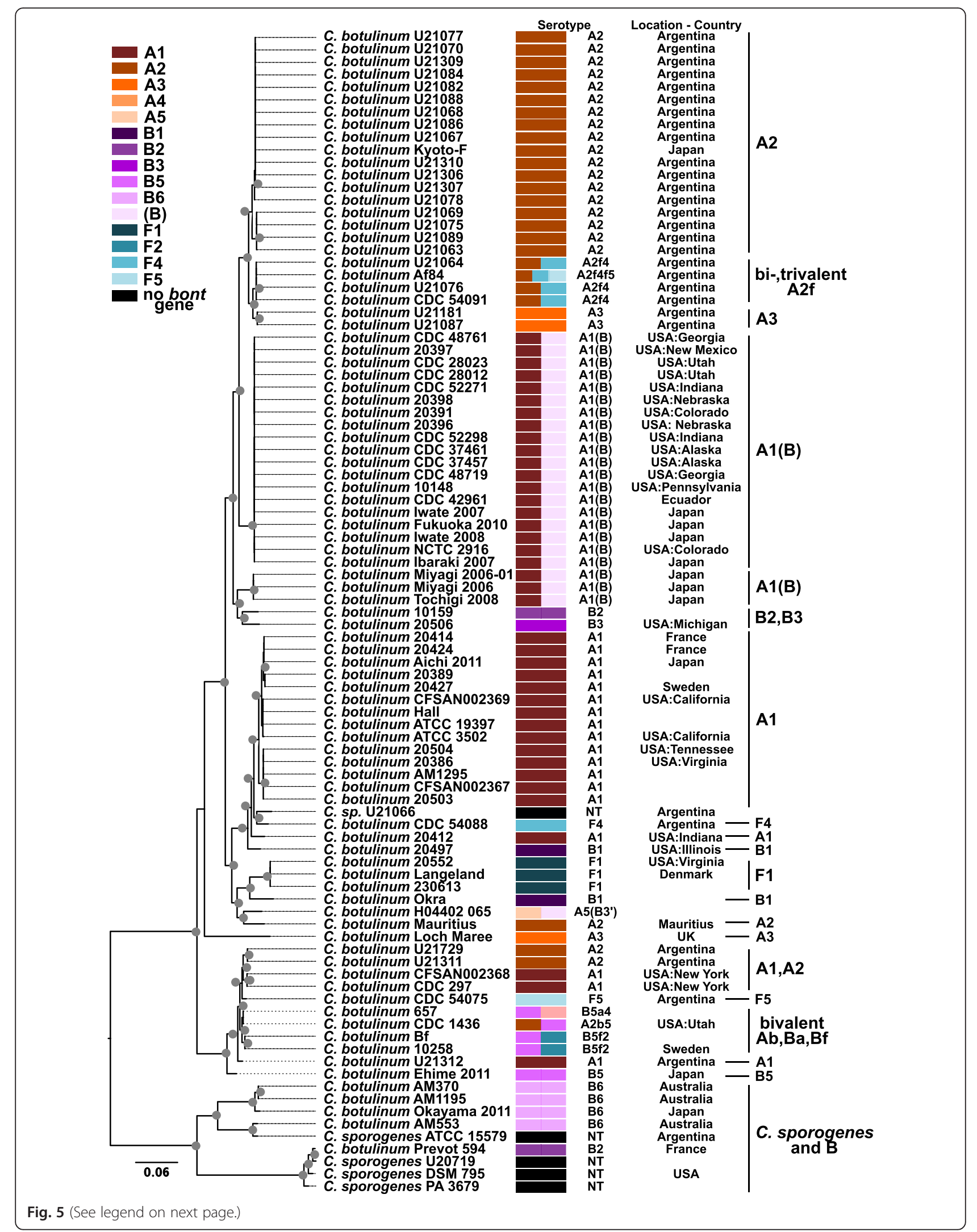


(See figure on previous page.)

Fig. 5 Group I core genome phylogeny. Core genome phylogeny of C. botulinum Group I inferred with RAxML v8.1.1 [63] using the ASC_GTRGAMMA model on an alignment of 200,641 core genome SNPs produced with NASP [61] using C. botulinum strain Kyoto-F [GenBank:CP001581] as a reference genome. The consistency index is 0.57 , and the retention index is 0.91 . Gray circles indicate bootstrap values over $95 \%$. The phylogeny was rooted with the clade that includes Clostridium sporogenes and C. botulinum B serotypes (bottom of Figure) using FigTree [55]. Strains are color-coded by botulinum neurotoxin serotype. Additional information regarding bont gene cluster characteristics is included in Table 1

\section{SNP and homoplasy density in C. botulinum Groups I and II}

Recombination is a common evolutionary process [90] that has been detected among C. botulinum strains [21] and may impact accurate phylogenetic inference. Regions of the genome with high proportions of homoplasious SNPs (shared SNP alleles found in different lineages of a phylogeny not inherited from a common ancestor) may indicate regions of recombination [91]. For Groups I and II, parsimony informative SNPs (sites at which at least two SNP alleles are present in at least two genomes) and putative recombination, indicated by regions of the genome with high proportions of homoplasious SNPs, appear to be distributed throughout the core genome (Fig. 7a and c). A comparison of the histograms of homoplasy density ratio values for $1 \mathrm{~kb}$ segments of the core genomes for Groups I and II (Fig. 7b and d) suggests that recombination is more prevalent in Group I strains as compared to Group II strains. It should be noted that fewer Group II strains were evaluated and the underlying reasons for the apparent difference in the prevalence of recombination in each Group is unknown. Additionally, the approach to detect recombination used here does not address potential recombination (or lack thereof) between or within specific clades of the Group I or Group II phylogenies. Additional analyses could provide more information about recombined regions in BoNT-producing clades (e.g. are certain types of genes more impacted by recombination events).

\section{Gene content of $\boldsymbol{C}$. botulinum Groups I and II}

LS-BSR [74], a tool for comparing the relatedness of coding sequences among genomes, was used to evaluate the genetic content of Group I and II strains and to cluster strains based upon coding sequence similarity (Additional file 8: Figure S5). Dendrograms of Group I and II strains constructed by clustering BSR values with an average linkage algorithm (this method considers the entire pan-genome for each Group) show similar overall strain clustering patterns as the core genome phylogenies for both Groups I and II. These clustering patterns indicate that clades identified by core genome phylogenies share coding sequence similarity. Future research could investigate potential functional roles of different clades and could identify marker genes for different clades.

The LS-BSR tool was also applied to investigate the presence or absence of cold shock protein genes in Group II assemblies. Söderholm and colleagues [92] demonstrated that cold shock protein genes, which are commonly found in microbes, were absent from three Group II BoNT/E genome assemblies but were present in the Group II strain Eklund 17B (BoNT/B) genome. Although Group II strains have been shown to grow and produce toxins at low temperatures [93], the LS-BSR approach demonstrated that homologs of cold shock protein genes found in other C. botulinum genomes are absent from Group II BoNT/E genomes, while cold

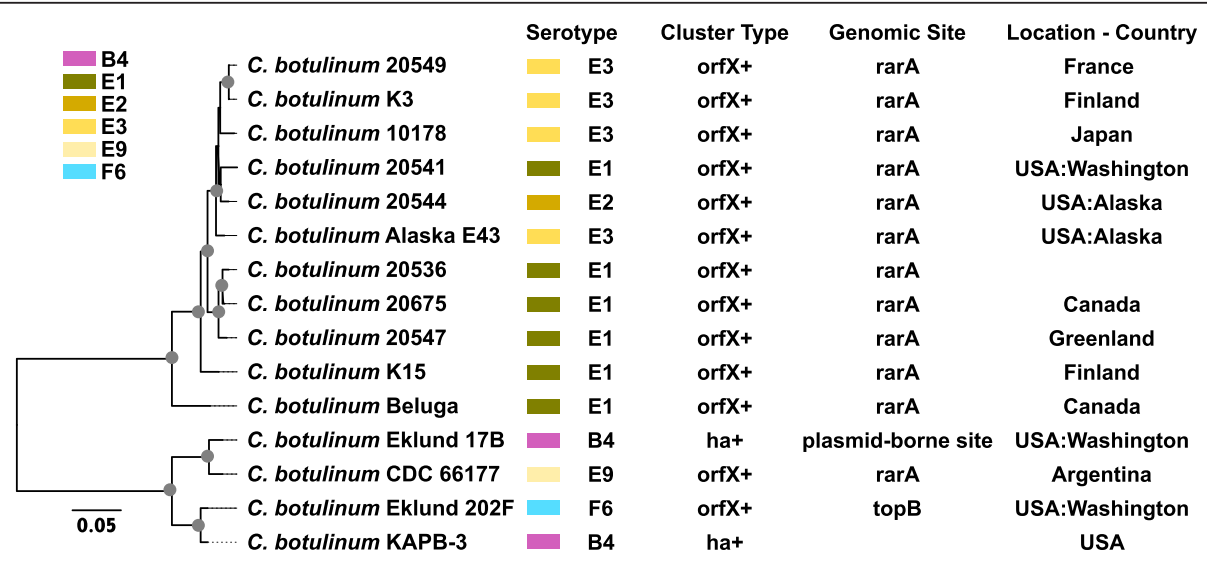

Fig. 6 Group II core genome phylogeny. Core genome phylogeny of C. botulinum Group II inferred with RAxML v8.1.1 [63] using the ASC_GTRGAMMA model on an alignment of 197,688 core genome SNPs produced with NASP [61] using C. botulinum Eklund 17B [GenBank:CP001056, CP001057] as a reference genome. The consistency index is 0.81 , and the retention index is 0.90 . Gray circles indicate bootstrap values over $95 \%$. The phylogeny was rooted with the clade that includes strains Eklund 202F, KAPB-3, Eklund 17B and CDC 66177 using FigTree [55]. Strains are color-coded by botulinum neurotoxin serotype 


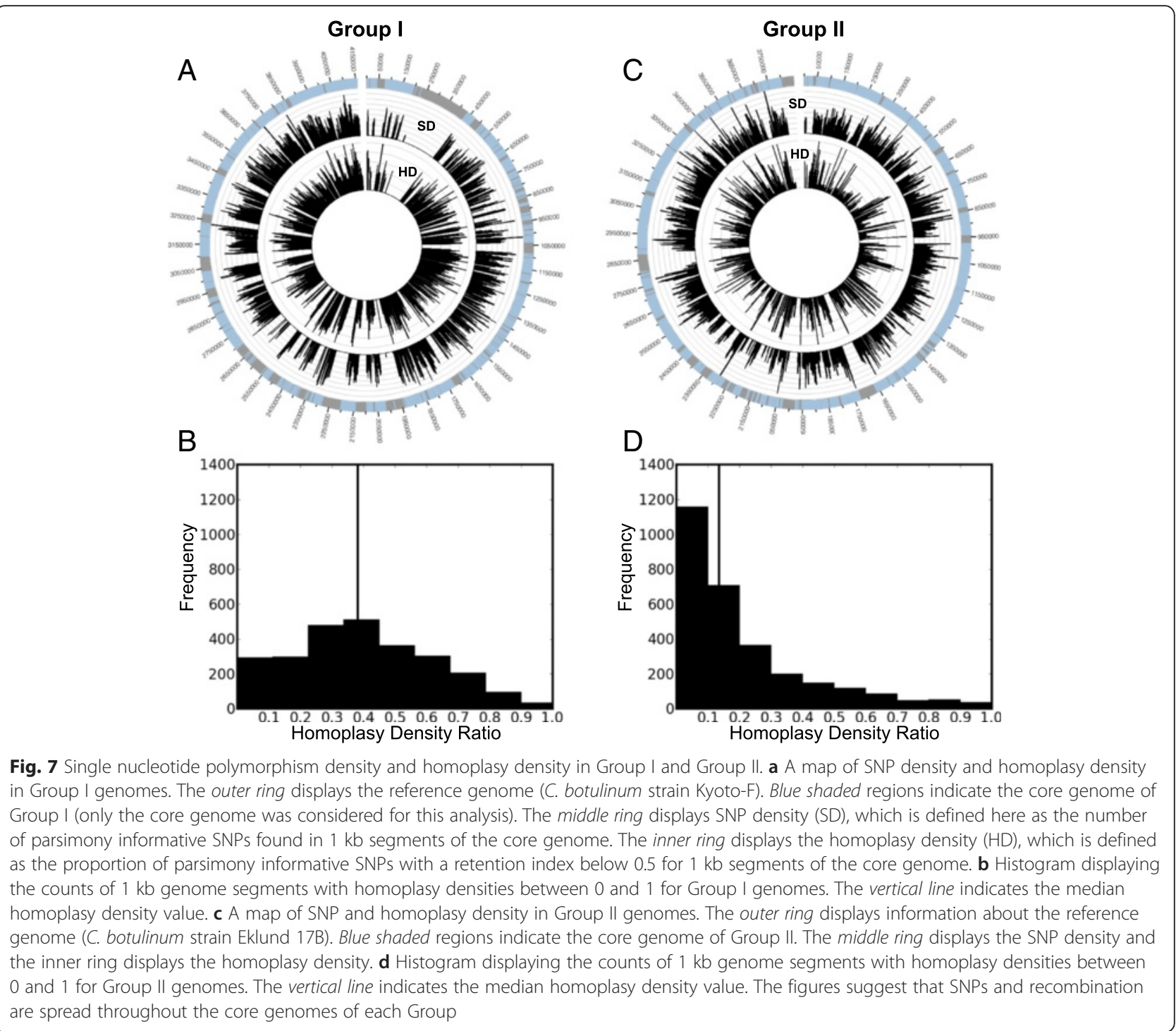

shock protein genes are present in the genomes falling into the Group II clade including BoNT/B,/E9 and/F genomes (Eklund 17B, KAPB-3, CDC 66177 and Eklund 202F).

\section{bont gene cluster analyses}

The bont genes from each of the newly sequenced strains were evaluated to determine if there were any new variants or subtypes. A maximum likelihood phylogenetic tree of bont gene sequences extracted from genome assemblies and previously published bont gene sequences is presented (Additional file 9: Figure S6). Subtype-specific clades can be seen for serotypes. Recently, new bont subtypes have been identified for serotypes A [94] and E [95, 96], and an unusual toxin type ( $\mathrm{H}$ or $\mathrm{F} / \mathrm{A})$ was identified in an isolate from an infant botulism case $[4,5]$. However, bont gene sequences from newly sequenced strains in this study are closely related
(>99 \% BLAST identity) to known bont subtype gene sequences, indicating that novel bont subtypes were not discovered in the newly sequenced strains.

Botulinum neurotoxin genes are associated with two gene cluster types $(h a+$ and orf $X+)$ that appear to be located at specific locations in the chromosome or within a plasmid [3]. Information regarding the bont type and putative bont gene cluster location for draft genome assemblies is included in Table 1. The presence of a marker gene $(P L-6)$ for identifying bont-containing plasmids [80] in all genome assemblies was determined with BLAST. The PL-6 gene was present in genome assemblies of published Group I strains known to have plasmids containing bont genes (Af84, Bf, CDC 657, CDC 1436, CDC 54075, Loch Maree, Okra, Prevot 594) as well as newly sequenced Group I strains with bont genes putatively located on plasmids (10258, AM370, 
AM553 and AM1195 - all bont/B strains). The PL-6 gene was not identified in assemblies that do not contain bont-containing plasmids.

The putative location of toxin gene clusters in the newly sequenced strains was examined and compared to previously published BoNT-producing strain genomes (Table 1). The bont/A1 genes in ha+ gene clusters have putative chromosomal locations and are associated with oppA/brnQ operons. The orf X+ bont/ A1 gene in strain U21312 and in serotype A1(B) strains is located within the chromosome and associated with the $\operatorname{ars} C$ operon, which is also the case in the published orf $X+$ bont/A1 strain CDC 297 [1] and serotype A1(B) strain NCTC 2916 [97]. The bont/A2 genes in newly sequenced genomes have putative chromosomal locations and are associated with $\operatorname{orf} X+$ gene clusters and ars $C$ operons, which are common traits for bont/A2 genomes $[9,98]$. This is in contrast to the bivalent strain CDC 1436 where the bont/A2 gene and a bont/B5 gene are within a plasmid [1]. Two Argentinian bont/A3 strains (U21087 and U21181) and the Loch Maree strain contain orf $X+$ bont gene cluster types. The bont/A3 gene of the Loch Maree strain is located within a plasmid while the location of the bont/A3 gene of the newly sequenced serotype A3 strains is unclear. All of the bont/B genes in newly sequenced and published Group I and II strains are putatively found in ha+ gene clusters. Interestingly, while the bont/B1 gene in the published Okra strain is located on a plasmid [88], the bont/B1 gene in newly sequenced strain 20497 has a putative chromosomal location. In contrast to the plasmid location of the bont/B2 gene in strain Prevot 594 [1], the bont/B2 gene in strain 10159 and strain IBCA1-7060 [4] and bont/B3 gene in 20506 have putative chromosomal locations. The bont/B6 genes in the newly sequenced Australian strains (AM370, AM553, AM1195) have putative plasmid locations, which is consistent with published bont/B6 subtypes from Japan [50]. The bont/F genes of newly sequenced Group I strains are found in $\operatorname{orf} X+$ gene clusters, which is consistent with previously published genomes $[9,98,99]$. All bont/F1 genes in strains analyzed in this study are associated with $\operatorname{ars} C$ operons and have chromosomal locations. Toxin genes in Group I bivalent bont/Af strains are associated with orf $X+$ gene clusters, and the bont/A2 and bont/f4 genes have putative chromosomal locations. The bont/f5 gene of the trivalent strain Af84 is located within a plasmid [98]. Bivalent bont/Ab,/Ba and/Bf strains in Group I have bont genes putatively located on plasmids. All the bont/E genes in the Group II strains analyzed here are associated with $\operatorname{orf} X+$ gene clusters and $\operatorname{rar} A$ operons and have putative chromosomal locations.

\section{Discussion}

Diverse members of the Clostridia produce potent botulinum neurotoxins that cause botulism, a flaccid paralysis that affects humans, other mammals, birds and fish. These microbes are of interest for public health and biodefense reasons [100]. Horizontal gene transfer, insertion and recombination events have been documented in BoNT-producing clostridia [1,7-10], resulting in variation in botulinum neurotoxins, toxin gene clusters and the genomic backgrounds in which the same toxin type or subtype are found. Thus, whole genome sequencing of Clostridia strains was conducted to provide insight into toxin gene cluster characterisitics, genomic diversity, phylogenetic relationships and global distribution of BoNT-producing Clostridia. Genomic sequence data and draft genome assemblies were generated for 59 Clostridia strains from six continents providing a resource to the research community. These data were compared to publicly available whole genome sequence data using multiple methods. Group designation of BoNTproducing strains was achieved with $16 \mathrm{~S}$ rRNA gene sequence phylogenies and confirmed by phylogenies that included larger swaths of the genome: a phylogeny of concatenated marker genes and a referenceindependent, SNP-based phylogeny. While the topologies of the three different phylogenies show some variation, the Group designations of strains are consistent among all three methods. The concatenated marker genes and reference-independent, SNP-based phylogenies demonstrate the diversity within the BoNTproducing Groups I, II and III that has been described with other methods [7, 11, 15, 81, 84].

Core genome phylogenies of Group I and II strains (Figs. 5 and 6) illustrate the phylogenetic diversity within each Group. Homoplasy is distributed throughout the Group I and II core genomes (Fig. 7), which suggests a history of recombination and could affect accurate phylogenetic inference. However, the distinct groupings as well as the diversity of Group I and II strains are apparent. Clustering of genomes based upon gene content (BSR values) produces similar groupings of strains as the core genome phylogenies for both Groups. ANIm values reveal the genomic diversity within both Groups I and II indicating that multiple species, subspecies or genomovars are likely present in each Group [86]. Weigand and colleagues [84] recently delineated Group I C. botulinum and C. sporogenes strains using similar comparative genomic techniques. The diversity within BoNT-producing Clostridia should be considered when studying these microbes. For example, appropriate strains must be chosen for conducting microbiological challenge tests used for evaluating the risk of BoNT-producing Clostridia or botulinum neurotoxins contaminating food [101-103]. 
The core genome phylogenies of Group I and II strains also illustrate the diversity of genomic backgrounds that express the same toxin type or subtype. Examples of different genomic backgrounds containing the same toxin type/subtype are abundant in Group I. Subtype bont/A1 strains are found in multiple clades. Argentinian bont/ A2 strains and bont/A2 strain Kyoto-F cluster distantly from the Mauritius bont/A2 strain and two other Argentinian bont/A2 strains. Two rare bont/A3 strains from Argentina cluster distantly from the bont/A3 Loch Maree strain from Scotland. A clade of bivalent bont/Af strains is distantly related to a clade of bivalent strains containing bont/A, bont/B and bont/F genes indicating the diversity of genomic backgrounds containing different combinations of these three toxin types. Two clades of bont/A1(B) strains are distinguished: one clade with isolates from Ecuador, Japan and the US and the other clade with isolates from Japan. Subtype bont/B1 and/B2 strains in which the bont genes have putative chromosomal locations are not closely related to previously sequenced bont/B1 and/B2 strains in which the bont genes are located on plasmids [1, 88]. This distribution of toxin types and subtypes throughout the Group I phylogeny suggests a history of horizontal gene transfer.

This study includes BoNT-producing strains isolated from both environmental sources and human botulism cases from across the globe; however, whole genome sequence data for strains from many parts of the world are not available. Large geographical gaps are evident in much of Africa, Asia, and South America suggesting much additional diversity is unsampled and unknown. Diversity of both genomic backgrounds and toxin type is apparent in some geographic regions for which many genome assemblies are available. For example, Argentina is a major reservoir of many serotypes. Interestingly, many BoNT/A2 strains were identified in Argentina, and examination of the Group I core genome phylogeny illustrates the genomic diversity present in this country. While some subtypes have been reported only in certain locales (e.g. bont/A2f4 in Argentina [98, 104]), many subtypes and/or strains that are closely related by core genome comparisons are found in distant locations. For instance, BoNT/A1-producing strains are found throughout the world, and bont/A1(B) strains showing a close phylogenetic relationship are found in Ecuador, Japan and the United States. Patterns of global serotype and genomic background distribution are not apparent from the analyses presented in this study. Understanding the global distribution of BoNT-producing strains is complex as these Clostridia are spore-forming microbes capable of persisting in an environment and can be distributed by a number of dispersal processes. However, a better understanding of the phylogeography of BoNT-producing strains and of the ability for strain attribution in events such as public health emergencies (e.g. foodborne outbreaks) may be possible through additional whole genome sequencing efforts (including producing complete genomes representing the diversity of BoNT-producing strains).

The comparative genomic techniques used in this study are capable of differentiating BoNT-producing Clostridia (including strains of the same serotype and from similar geographic locations) and provide a framework for the study of these toxin-producing microbes (e.g. investigating horizontal gene transfer and phylogeography). As more whole genome sequence data for BoNT-producing strains become available our understanding of the diversity and distribution of these microbes has expanded. Recent whole genome sequencing of serotype $\mathrm{E}$ strains revealed information on stress response [92] and new bont/E toxin variants [95], and whole genome sequencing has identified bont/B6 strains in Australia that are closely related to bont/B6 strains previously reported in Japan [50]. Future research utilizing the growing number of whole genome sequenced strains will further our understanding of these microbes and potentially aid the development of diagnostics and treatments. For instance, comparative genomics may aid in the identification of marker genes capable of identifying strains within Groups (or specific clades in each Group) more rapidly than methods relying on cultivation and subsequent whole genome sequencing.

\section{Conclusions}

Whole genome sequence analyses of Clostridia species illustrate the diversity of botulinum-neurotoxinproducing strains and the plasticity of the genomic backgrounds in which bont genes are found. Core genome phylogenies are a powerful tool for differentiating BoNT-producing strains and providing a framework for the study of these bacteria. As more BoNT-producing strains are whole genome sequenced, our understanding of the genomic diversity of microbes capable of producing potent botulinum neurotoxins will continue to expand.

\section{Availability of supporting data}

Whole genome sequence data in support of the results of this article have been deposited in the NCBI Sequence Read Archive (BioProject ID PRJNA286797, study accession number SRP059640). Draft genome assemblies have been deposited in the NCBI WGS database; accession numbers for draft genome assemblies are included in Table 1 and Additional file 1: Table S1. Phylogenetic data 
is deposited in the TreeBase database (Study 18872) and https://github.com/chawillia/phylogenetic_data_2016.

\section{Additional files}

Additional file 1: Table S1. Information regarding newly sequenced strains analyzed in this study. Additional information regarding newly sequenced strains. (XLSX $22 \mathrm{~kb}$ )

Additional file 2: Table S2. Information regarding publicly available genomes examined in this study. Table of information regarding genomes included in this study. (XLSX $27 \mathrm{~kb}$ )

Additional file 3: Table S3. Table of all pairwise ANIm values. Table of pairwise ANIm values computed with JSpecies [72]. (XLSX 168 kb)

Additional file 4: Figure S1. Core genome phylogenies of Group I strains. A) Core genome phylogeny of C. botulinum Group I strains inferred with RAxML [63] using the ASC_GTRGAMMA model on an alignment of 182,200 core genome SNPs produced with NASP [61] using C. sporogenes ATCC 15579 [GenBank:ABKW00000000] as the reference genome. The consistency index is 0.56 , and the retention index is 0.91 (computed with the R package phangorn). B) Core genome phylogeny of Group I strains inferred with FastTree2 [54] on a 1780-character core SNP matrix generated with kSNP [59]. The consistency index is 0.64 , and the retention index is 0.93 . The phylogenies were rooted with the clade that includes C. sporogenes and C. botulinum bont/B serotypes (bottom of Figure). Gray circles indicate bootstrap values over $95 \%$. While there are small variations in the phylogenies generated with different methods (Fig. 5 and Additional file 4: Figure S1), the overall topology of the Group I tree appears robust. The pairwise overall topological scores computed by Compare2Trees [69] range from 80 to $86 \%$ for the phylogenies presented in Fig. 5 and (Additional file 4: Figure S1). (PDF 59 kb)

Additional file 5: Figure S2. Group I concatenated MLST gene phylogeny. Phylogeny of aligned (MUSCLE [57]) and concatenated MLST genes for Group I genomes inferred with FastTree2 [54]. The MLST profile included aceK, aroE, hsp60, $m d h$, oppB, recA and rpoB [21]. Taxa labeled ST are 83 sequence types available from PubMLST [77, 78]. Investigation of the concatenated MLST gene phylogeny suggests that diverse BoNTproducing strains have yet to be whole genome sequenced. (PDF $31 \mathrm{~kb}$ )

Additional file 6: Figure S3. Core genome phylogenies of Group II strains. A) Core genome phylogeny of C. botulinum Group II strains inferred with RAXML [63] using the ASC_GTRGAMMA model on an alignment of 200,276 core genome SNPs produced with NASP [61] using C. botulinum strain Alaska E43 [GenBank:CP001078] as the reference. The consistency index is 0.81 , and the retention index is 0.90 (computed with the $\mathrm{R}$ package phangorn). B) Core genome phylogeny of Group II C. botulinum strains inferred with FastTree2 [54] on a 35,382-character core SNP matrix generated with kSNP [59]. The consistency index is 0.83 , and the retention index is 0.90 . The phylogenies were rooted with the clade that includes strains Eklund 202F, KAPB-3, Eklund 17B and CDC 66177. Gray circles indicate bootstrap values over $95 \%$. While there are small variations in the phylogenies generated with different methods (Fig. 6 and Additional file 6: Figure S3), Group II strains are separated into two distinct clades. The pairwise overall topological scores computed by Compare2Trees [69] range from 93 to $100 \%$. (PDF 35 kb)

Additional file 7: Figure S4. Group II concatenated MLST gene phylogeny. Phylogeny of aligned (MUSCLE [57]) and concatenated MLST genes for Group II genomes inferred with FastTree2 [54]. The MLST profile included $16 \mathrm{~S} r R N A$ genes, atpD, guaA, gyrB, ilvD, lepA, mutL, oppB, pta, pyc, recA, rpoB, trpB and tuf [22]. Taxa labeled C. botulinum are WGS samples. Taxa labeled with single-word name include serotype $\mathrm{E}$ strains for which the MLST genes were sequenced by MacDonald and colleagues [22]. (PDF $37 \mathrm{~kb}$ )

Additional file 8: Figure S5. Dendrograms clustering Group I and Group II strains by BSR values. A) A dendrogram generated by clustering Group I strains by BSR values with an average linkage method in MeV [76]. B) A dendrogram generated by clustering Group II strains by BSR values with an average linkage method in MeV [76]. (PDF $41 \mathrm{~kb}$ )
Additional file 9: Figure S6. bont gene phylogeny. A phylogeny inferred with FastTree2 [54] on a nucleotide alignment (MUSCLE [57]) of botulinum neurotoxin genes. Gray circles indicate bootstrap values over $90 \%$. The tree was rooted with tetanus toxin gene sequences in FigTree [55]. (PDF 54 kb)

\section{Abbreviations}

AFLP: amplified fragment length polymorphism; ANIm: average nucleotide identity (MUMmer method); BLAST: basic local alignment search tool; BoNT: botulinum neurotoxin; bont: botulinum neurotoxin gene; $C$. botulinum: Clostridium botulinum; DNA: deoxyribonucleic acid; IS: insertion sequence; LS-BSR: large-scale BLAST score ratio; MeV: MultiExperiment viewer; MLST: multi-locus sequence typing; NCBI: National Center for Biotechnology Information; PCR: polymerase chain reaction; PFGE: pulsedfield gel electrophoresis; rRNA: ribosomal ribonucleic acid; SNP: single nucleotide polymorphism; ST: sequence type; WGS: whole genome sequence.

\section{Competing interests}

The authors declare they have no competing interests.

\section{Authors' contributions}

KKH, JTF, PK, JWS, TJS, CHDW designed the study. CHDW, JWS, KKH, GX analyzed the data. RAF, ML, HK, KKH, TJS provided bacterial strains or DNA. TJS provided the provenance of many strains. CHDW and KKH drafted the initial manuscript. All authors read, revised, and approved the final manuscript.

\section{Acknowledgements}

The work was supported by the U.S. Department of Homeland Security Science and Technology Directorate via awards HSHQDC-10-C-00139 and DHS-2010-ST-108-000019. CHDW was supported by funding from Arizona's Technology Research Initiative Fund. Some of the computational analyses were run on Northern Arizona University's Monsoon computing cluster, also funded by Arizona's Technology and Research Initiative Fund. The authors would like to thank Dr. C. Shone of Public Health England, Salisbury, UK for his kind gift of DNA from the Mauritius bont/A2 strain, and Dr. J. Rood of Monash University, Melbourne, Australia for providing C. botulinum strains for analyses. Opinions, interpretations, conclusions and recommendations are those of the authors and not necessarily endorsed by the U.S. Army.

\section{Author details}

${ }^{1}$ Center for Microbial Genetics and Genomics, Northern Arizona University, Flagstaff, AZ 86011, USA. ${ }^{2}$ Molecular and Translational Sciences Division, United States Army Medical Research Institute of Infectious Diseases, Fort Detrick, MD 21702, USA. ${ }^{3}$ Bioscience Division, Los Alamos National Laboratory, Los Alamos, NM 87545, USA. ${ }^{4}$ Theoretical Division, Los Alamos National Laboratory, Los Alamos, NM 87545, USA. ${ }^{5}$ Medical Countermeasures Technology, United States Army Medical Research and Material Command, United States Army Medical Research Institute of Infectious Diseases, Fort Detrick, MD 21702 , USA. 'Área Microbiología, Departamento de Patología, Facultad de Ciencias Médicas, Universidad Nacional de Cuyo, Centro Universitario, (5500) Mendoza, Argentina. ${ }^{7}$ Department of Food Hygiene and Environmental Health, University of Helsinki, Helsinki, Finland. ${ }^{8}$ Present Address: Department of Molecular, Cellular and Biomedical Sciences, University of New Hampshire, Durham, NH 03824, USA.

\section{Received: 30 September 2015 Accepted: 18 February 2016}

Published online: 03 March 2016

\section{References}

1. Smith TJ, Hill KK, Xie G, Foley BT, Williamson CHD, Foster JT, et al. Genomic sequences of six botulinum neurotoxin-producing strains representing three Clostridial species illustrate the mobility and diversity of botulinum neurotoxin genes. Infect Genet Evol. 2015;30:102-13.

2. Peck MW. Biology and genomic analysis of Clostridium botulinum. Adv Microb Physiol. 2009;55:183-320.

3. Hill KK, Smith TJ. Genetic diversity within Clostridium botulinum serotypes, botulinum neurotoxin gene clusters and toxin subtypes. In: Rummel A, Binz T, editors. Botulinum neurotoxins. Springer: Berlin Heidelberg; 2013. p. 1-20.

4. Dover N, Barash JR, Hill KK, Xie G, Arnon SS. Molecular characterization of a novel botulinum neurotoxin type H gene. J Infect Dis. 2014;209:192-202. 
5. Kalb SR, Baudys J, Raphael BH, Dykes JK, Lúquez C, Maslanka SE, et al. Functional characterization of botulinum neurotoxin serotype $\mathrm{H}$ as a hybrid of known serotypes F and A (BoNT F/A). Anal Chem. 2015;87:3911-7.

6. Collins MD, East AK. Phylogeny and taxonomy of the food-borne pathogen Clostridium botulinum and its neurotoxins. J Appl Micro. 1998;84:5-17.

7. Hill KK, Smith TJ, Helma CH, Ticknor LO, Foley BT, Svensson RT, et al. Genetic diversity among botulinum neurotoxin-producing Clostridial strains. J Bacteriol. 2007;189:818-32.

8. Moriishi K, Koura M, Abe N, Fujii N, Fujinaga Y, Inoue K, et al. Mosaic structures of neurotoxins produced from Clostridium botulinum types $\mathrm{C}$ and D organisms. Biochim Biophys Acta. 1996;1307:123-6.

9. Hill KK, Xie G, Foley BT, Smith TJ, Munk AC, Bruce D, et al. Recombination and insertion events involving the botulinum neurotoxin complex genes in Clostridium botulinum types $\mathrm{A}, \mathrm{B}, \mathrm{E}$ and $\mathrm{F}$ and Clostridium butyricum type $\mathrm{E}$ strains. BMC Biol. 2009;7:66.

10. Skarin $H$, Segerman B. Horizontal gene transfer of toxin genes in Clostridium botulinum. Mob Genet Elements. 2011:1:213-5.

11. Skarin H, Håfström T, Westerberg J, Segerman B. Clostridium botulinum Group III: a group with dual identity shaped by plasmids, phages and mobile elements. BMC Genomics. 2011;12:185.

12. Smith TJ, Hill KK, Raphael BH. Historical and current perspectives on Clostridium botulinum diversity. Res Microbiol. 2014;166:290-302.

13. Sebaihia M, Peck MW, Minton NP, Thomson NR, Holden MTG, Mitchell WJ, et al. Genome sequence of a proteolytic (Group I) Clostridium botulinum strain Hall A and comparative analysis of the Clostridial genomes. Genome Res. 2007;17:1082-92.

14. Lindström M, Hinderink $K$, Somervuo P, Kiviniemi $K$, Nevas $M$, Chen $Y$, et al. Comparative genomic hybridization analysis of two predominant Nordic Group I (proteolytic) Clostridium botulinum type B clusters. Appl Environ Microb. 2009:75:2643-51.

15. Stringer SC, Carter AT, Webb MD, Wachnicka E, Crossman LC, Sebaihia M, et al. Genomic and physiological variability within Group II (non-proteolytic) Clostridium botulinum. BMC Genomics. 2013;14:333

16. Keto-Timonen $\mathrm{R}$, Nevas M, Korkeala $\mathrm{H}$. Efficient DNA fingerprinting of Clostridium botulinum types $A, B, E$, and $F$ by amplified fragment length polymorphism analysis. Appl Environ Microb. 2005;71:1148-54.

17. Dabritz HA, Hill KK, Barash JR, Ticknor LO, Helma CH, Dover N, et al. Molecular epidemiology of infant botulism in California and elsewhere, 1976-2010. J Infect Dis. 2014:210:1711-22.

18. Lin WJ, Johnson EA. Genome analysis of Clostridium botulinum type A by pulsed-field gel electrophoresis. Appl Environ Microb. 1995;61:4441-7.

19. Hielm S, Björkroth J, Hyytiä E, Korkeala H. Genomic analysis of Clostridium botulinum Group II by pulsed-field gel electrophoresis. Appl Environ Microb. 1998;64:703-8.

20. Franciosa G, Maugliani A, Scalfaro C, Aureli P. Evidence that plasmid-borne botulinum neurotoxin type $B$ genes are widespread among Clostridium botulinum serotype B strains. PLoS ONE. 2009;4:e4829.

21. Jacobson MJ, Lin G, Whittam TS, Johnson EA. Phylogenetic analysis of Clostridium botulinum type A by multi-locus sequence typing. Microbiology. 2008;154:2408-15

22. Macdonald TE, Helma CH, Shou Y, Valdez YE, Ticknor LO, Foley BT, et al. Analysis of Clostridium botulinum serotype $E$ strains by using multilocus sequence typing, amplified fragment length polymorphism, variablenumber tandem-repeat analysis, and botulinum neurotoxin gene sequencing. Appl Environ Microb. 2011;77:8625-34.

23. McCallum N, Gray TJ, Wang Q, Ng J, Hicks L, Nguyen T, et al. Genomic epidemiology of Clostridium botulinum isolates from temporally related cases of infant botulism in New South Wales, Australia. J Clin Microbiol. 2015;53:2846-53.

24. Midura TF, Nygaard GS, Wood RM, Bodily HL. Clostridium botulinum type F: isolation from venison jerky. Appl Microbiol. 1972;24:165-7.

25. Hyytiä $\mathrm{E}$, Hielm S, Korkeala H. Prevalence of Clostridium botulinum type $\mathrm{E}$ in Finnish fish and fishery products. Epidemiol Infect. 1998;120:245-50.

26. Brett MM. A case of infant botulism with a possible link to infant formula milk powder: evidence for the presence of more than one strain of Clostridium botulinum in clinical specimens and food. J Med Microbiol. 2005:54:769-76.

27. Smith LD. The occurrence of Clostridium botulinum and Clostridium tetani in the soil of the United States. Health Lab Sci. 1978;15:74-80.

28. Lúquez C, Bianco MI, de Jong LIT, Sagua MD, Arenas GN, Ciccarelli AS, et al. Distribution of botulinum toxin-producing Clostridia in soils of Argentina. Appl Environ Microb. 2005;71:4137-9.
29. Nevas M, Hielm S, Lindström M, Horn H, Koivulehto K, Korkeala H. High prevalence of Clostridium botulinum types $\mathrm{A}$ and $\mathrm{B}$ in honey samples detected by polymerase chain reaction. Int J Food Microbiol. 2002;72:45-52.

30. Midura TF, Snowden S, Wood RM, Arnon SS. Isolation of Clostridium botulinum from honey. J Clin Microbiol. 1979;9:282-3.

31. Schocken-Iturrino RP, Carneiro MC, Kato E, Sorbara JO, Rossi OD, Gerbasi LE. Study of the presence of the spores of Clostridium botulinum in honey in Brazil. FEMS Immunol Med Microbiol. 1999;24:379-82.

32. Leclair D, Farber JM, Doidge B, Blanchfield B, Suppa S, Pagotto F, et al. Distribution of Clostridium botulinum Type E strains in Nunavik, Northern Quebec, Canada. Appl Environ Microb. 2013;79:646-54.

33. Bianco MI, Lúquez C, de Jong LIT, Fernández RA. Presence of Clostridium botulinum spores in Matricaria chamomilla (chamomile) and its relationship with infant botulism. Int J Food Microbiol. 2008;121:357-60.

34. Eklund MW, Poysky FT. Distribution of Clostridium botulinum on the Pacific Coast of the United States. In: Herzberg M, editor. Proceedings of the First U.S. - Japan Conference on Toxic Microorganisms. Honolulu, HI; 1968. p. 304-308.

35. Sahl JW. UGAP. https://github.com/jasonsahl/UGAP/. Accessed 23 April 2014.

36. Bolger AM, Lohse M, Usadel B. Trimmomatic: a flexible trimmer for Illumina sequence data. Bioinformatics. 2014;30:2114-20.

37. Nikolenko SI, Korobeynikov Al, Alekseyev MA. BayesHammer: Bayesian clustering for error correction in single-cell sequencing. BMC Genomics. 2013;14 Suppl 1:S7.

38. Bankevich A, Nurk S, Antipov D, Gurevich AA, Dvorkin M, Kulikov AS, et al. SPAdes: a new genome assembly algorithm and its applications to singlecell sequencing. J Comput Biol. 2012;19:455-77.

39. Li W, Godzik A. Cd-hit: a fast program for clustering and comparing large sets of protein or nucleotide sequences. Bioinformatics. 2006;22:1658-9.

40. Li H, Durbin R. Fast and accurate short read alignment with BurrowsWheeler transform. Bioinformatics. 2009;25:1754-60.

41. Li H. Aligning sequence reads, clone sequences and assembly contigs with BWA-MEM. arXiv preprint arXiv:1303.3997. 2013.

42. DePristo MA, Banks E, Poplin R, Garimella KV, Maguire JR, Hartl C, et al. A framework for variation discovery and genotyping using next-generation DNA sequencing data. Nat Genet. 2011:43:491-8.

43. Walker BJ, Abeel T, Shea T, Priest M, Abouelliel A, Sakthikumar S, et al. Pilon: An Integrated Tool for Comprehensive Microbial Variant Detection and Genome Assembly Improvement. PLoS ONE. 2014;9(11):e112963.

44. Tsai IJ, Otto TD, Berriman M. Improving draft assemblies by iterative mapping and assembly of short reads to eliminate gaps. Genome Biol. 2010;11:R41.

45. Altschul SF, Gish W, Miller W, Myers EW, Lipman DJ. Basic local alignment search tool. J Mol Biol. 1990:215:403-10.

46. Wattam AR, Abraham D, Dalay O, Disz TL, Driscoll T, Gabbard JL, et al. PATRIC, the bacterial bioinformatics database and analysis resource. Nucl Acids Res. 2014:42:D581-91.

47. Benson DA, Karsch-Mizrachi I, Lipman DJ, Ostell J, Wheeler DL. GenBank Nucleic Acids Res. 2005;33 Suppl 1:D34-8

48. Seemann T. Prokka. rapid prokaryotic genome annotation. Bioinformatics. 2014;30:2068-9

49. Gurevich A, Saveliev V, Vyahhi N, Tesler G. QUAST: quality assessment tool for genome assemblies. Bioinformatics. 2013;29:1072-5.

50. Kenri T, Sekizuka T, Yamamoto A, Iwaki M, Komiya T, Hatakeyama T, et al. Genetic characterization and comparison of Clostridium botulinum isolates from botulism cases in Japan between 2006 and 2011. Appl Environ Microb. 2014:80:6954-64.

51. Edgar RC. Search and clustering orders of magnitude faster than BLAST. Bioinformatics. 2010;26:2460-1.

52. Nawrocki EP. Structural RNA homology search and alignment using covariance models. PhD thesis. St. Louis, Missouri: Washington University School of Medicine; 2009.

53. Schloss PD, Westcott SL, Ryabin T, Hall JR, Hartmann M, Hollister EB, et al. Introducing mothur: open-source, platform-independent, communitysupported software for describing and comparing microbial communities. Appl Environ Microb. 2009;75:7537-41.

54. Price MN, Dehal PS, Arkin AP. FastTree 2 - approximately maximumlikelihood trees for large alignments. PLoS ONE. 2010;5(3):e9490.

55. Rambaut A. FigTree. http://tree.bio.ed.ac.uk/software/figtree/. Accessed 9 July 2014.

56. Mende DR, Sunagawa S, Zeller G, Bork P. Accurate and universal delineation of prokaryotic species. Nat Meth. 2013;10:881-4. 
57. Edgar RC. MUSCLE: multiple sequence alignment with high accuracy and high throughput. Nucl Acids Res. 2004;32:1792-7.

58. Caporaso JG, Kuczynski J, Stombaugh J, Bittinger K, Bushman FD, Costello EK, et al. QIIME allows analysis of high-throughput community sequencing data. Nat Meth. 2010;7:335-6.

59. Gardner SN, Hall BG. When whole-genome alignments just won't work: KSNP V2 software for alignment-free SNP discovery and phylogenetics of hundreds of microbial genomes. PLOS ONE. 2013;8:e81760.

60. Kurtz S, Phillippy A, Delcher AL, Smoot M, Shumway M, Antonescu C, et al. Versatile and open software for comparing large genomes. Genome Biol. 2004;5:R12.

61. Sahl JW, Lemmer D, Travis J, Schupp J, Gillece J, Aziz M, et al. The Northern Arizona SNP Pipeline (NASP): accurate, flexible, and rapid identification of SNPs in WGS datasets. bioRxiv. 2016. doi: http://dx.doi.org/10.1101/037267.

62. McKenna A, Hanna M, Banks E, Sivachenko A, Cibulskis K, Kernytsky A, et al. The Genome Analysis Toolkit: a MapReduce framework for analyzing nextgeneration DNA sequencing data. Genome Res. 2010;20:1297-303.

63. Stamatakis A. RAxML Version 8: a tool for phylogenetic analysis and postanalysis of large phylogenies. Bioinformatics. 2014;30:1312-3.

64. Lewis PO. A likelihood approach to estimating phylogeny from discrete morphological character data. Syst Biol. 2001;50:913-25.

65. Stamatakis A, Hoover P, Rougemont J. A rapid bootstrap algorithm for the RAxML web servers. Syst Biol. 2008;57:758-71.

66. Pattengale ND, Alipour M, Bininda-Emonds ORP, Moret BME, Stamatakis A. How many bootstrap replicates are necessary? J Comput Biol. 2010;17:337-54

67. R Core Team. $R$ : a language and environment for statistical computing. Vienna: R Foundation for Statistical Computing; 2014.

68. Schliep KP. phangorn: phylogenetic analysis in R. Bioinformatics. 2011;27:592-3.

69. Nye TMW, Liò P, Gilks WR. A novel algorithm and web-based tool for comparing two alternative phylogenetic trees. Bioinformatics. 2006;22:117-9.

70. PAUP* SD. Phylogenetic Analysis Using Parsimony (*and Other Methods). Version 4. Sunderland: Sinauer Associates; 2003.

71. Krzywinski MI, Schein JE, Birol I, Connors J, Gascoyne R, Horsman D, et al Circos: an information aesthetic for comparative genomics. Genome Res. 2009;19(9):1639-45.

72. Richter M, Rosselló-Móra R. Shifting the genomic gold standard for the prokaryotic species definition. PNAS. 2009;106:19126-31.

73. Hunter JD. Matplotlib: a 2D graphics environment. Comput Sci Eng. 2007:9:90-5

74. Sahl JW, Caporaso JG, Rasko DA, Keim P. The large-scale blast score ratio (LS-BSR) pipeline: a method to rapidly compare genetic content between bacterial genomes. PeerJ. 2014;2:e332.

75. Kent WJ. BLAT-the BLAST-like alignment tool. Genome Res. 2002;12:656-64

76. Saeed Al, Bhagabati NK, Braisted JC, Liang W, Sharov V, Howe EA, et al. TM4 microarray software suite. Method Enzymol. 2006;411:134-93.

77. Jolley KA. Clostridium botulinum MLST Website. University of Oxford. http:// pubmlst.org/cbotulinum/. Accessed 7 Oct 2014.

78. Jolley KA, Maiden MC. BIGSdb: scalable analysis of bacterial genome variation at the population level. BMC Bioinformatics. 2010;11:595

79. Darling AE, Mau B, Perna NT. ProgressiveMauve: multiple genome alignment with gene gain, loss and rearrangement. PLOS ONE. 2010;5:e11147.

80. Raphael BH, Bradshaw M, Kalb SR, Joseph LA, Lúquez C, Barr JR, et al. Clostridium botulinum strains producing BoNT/F4 or BoNT/F5. Appl Environ Microb. 2014;80:3250-7.

81. Olsen JS, Scholz H, Fillo S, Ramisse V, Lista F, Trømborg AK, et al. Analysis of the genetic distribution among members of Clostridium botulinum Group I using a novel multilocus sequence typing (MLST) assay. J Microbiol Meth. 2014;96:84-91.

82. Gonzalez-Escalona N, Timme R, Raphael BH, Zink D, Sharma SK. Wholegenome single-nucleotide-polymorphism analysis for discrimination of Clostridium botulinum Group I strains. Appl Environ Microb. 2014;80:2125-32.

83. Raphael BH, Lautenschlager M, Kalb SR, de Jong LIT, Frace M, Lúquez C, et al. Analysis of a unique Clostridium botulinum strain from the Southern hemisphere producing a novel type $\mathrm{E}$ botulinum neurotoxin subtype. BMC Microbiol. 2012;12:245
84. Weigand MR, Pena-Gonzalez A, Shirey TB, Broeker RG, Ishaq MK, Konstantinidis KT, et al. Genome-based discrimination between Group I Clostridium botulinum and Clostridium sporogenes strains: implications for bacterial taxonomy. Appl Environ Microb. 2015:81(16):5420-9.

85. Rosselló-Móra R, Amann R. Past and future species definitions for Bacteria and Archaea. Syst Appl Microbiol. 2015;38:209-16.

86. Rosselló-Mora R, Amann R. The species concept for prokaryotes. FEMS Microbiol Rev. 2001;25:39-67.

87. Leighton G. Botulism and food preservation (the Loch Maree Tragedy). Glasgow: Collins and Sons; 1923.

88. Smith TJ, Hill KK, Foley BT, Detter JC, Munk AC, Bruce DC, et al. Analysis of the neurotoxin complex genes in Clostridium botulinum A1-A4 and B1 strains: BoNT/A3,/Ba4 and/B1 clusters are located within plasmids. PLoS ONE. 2007;2:e1271

89. Weedmark KA, Mabon P, Hayden KL, Lambert D, Domselaar GV, Austin JW, et al. Phylogenomic profiling of Clostridium botulinum Group ॥ isolates using whole genome sequence data. Appl Environ Microb. 2015;81(17):5938-48.

90. Didelot $\mathrm{X}$, Maiden MCJ. Impact of recombination on bacterial evolution. Trends Microbiol. 2010;18:315-22.

91. Driebe EM, Sahl JW, Roe C, Bowers JR, Schupp JM, Gillece JD, et al. Using whole genome analysis to examine recombination across diverse sequence types of Staphylococcus aureus. PLoS ONE. 2015;10:e0130955.

92. Söderholm H, Jaakkola K, Somervuo P, Laine P, Auvinen P, Paulin L, et al. Comparison of Clostridium botulinum genomes shows the absence of cold shock protein coding genes in type $\mathrm{E}$ neurotoxin producing strains. Botulinum J. 2013;2:189-207.

93. Graham AF, Mason DR, Maxwell FJ, Peck MW. Effect of $\mathrm{pH}$ and $\mathrm{NaCl}$ on growth from spores of non-proteolytic Clostridium botulinum at chil temperature. Lett Appl Microbiol. 1997;24:95-100.

94. Kull S, Schulz KM, Weisemann née Strotmeier J, Kirchner S, Schreiber T, Bollenbach A, et al. Isolation and functional characterization of the nove Clostridium botulinum neurotoxin A8 subtype. PLOS ONE. 2015;10:e0116381.

95. Weedmark KA, Lambert DL, Mabon P, Hayden KL, Urfano CJ, Leclair D, et al. Two novel toxin variants revealed by whole-genome sequencing of 175 Clostridium botulinum type E strains. Appl Environ Microb. 2014;80:6334-45.

96. Mazuet C, Sautereau J, Legeay C, Bouchier C, Bouvet P, Popoff MR. An atypical outbreak of food-borne botulism due to Clostridium botulinum types B and E from ham. J Clin Microbiol. 2015;53:722-6.

97. Jacobson MJ, Lin G, Raphael B, Andreadis J, Johnson EA. Analysis of neurotoxin cluster genes in Clostridium botulinum strains producing botulinum neurotoxin serotype A subtypes. Appl Environ Microb. 2008:74:2778-86.

98. Dover N, Barash JR, Hill KK, Davenport KW, Teshima H, Xie G, et al. Clostridium botulinum strain Af84 contains three neurotoxin gene clusters: bont/A2, bont/F4 and bont/F5. PLoS ONE. 2013;8:e61205.

99. Dover N, Barash JR, Hill KK, Detter JC, Arnon SS. Novel structural elements within the nonproteolytic Clostridium botulinum type F toxin gene cluster. Appl Environ Microb. 2011;77:1904-6.

100. Arnon SS, Schechter R, Inglesby TV, et al. Botulinum toxin as a biological weapon: medical and public health management. J Am Med Assoc. 2001;285:1059-70

101. Notermans S, in't Veld P, Wijtzes T, Mead GC. A user's guide to microbial challenge testing for ensuring the safety and stability of food products. Food Microbiol. 1993;10:145-57.

102. Doyle MP, Buchanan RL. Food Microbiology: Fundamentals and Frontiers. 4th ed. Washington DC: American Society for Microbiology Press; 2013.

103. Parker MD, Barrett PI, Shepherd J, Price LJ, Bull SD. Characterisation of nontoxigenic Clostridium spp. strains, to use as surrogates for non-proteolytic Clostridium botulinum in chilled food challenge testing. J Microbiol Meth. 2015:108:83-91.

104. Giménez DF, Ciccarelli AS. New strains of Clostridium botulinum subtype Af. Zentralbl Bakteriol Orig A. 1978;240:215-20. 